\title{
A systematic review of randomized controlled trials assessing phytochemicals and natural ingredients for skin and hair care
}

\author{
Samar Thiab ${ }^{\text {* }}$ (D), Nizar M. Mhaidat ${ }^{2}$, May Abu Taha ${ }^{1}$, Sarah Thiab $^{3}$, Somaya Koraysh ${ }^{3}$, Reem Abutayeh ${ }^{1}$, Iman Basheti ${ }^{1}$ \\ ${ }^{1}$ Faculty of Pharmacy, Applied Science Private University, College of Pharmacy, Qatar University, Amman, Jordan. \\ ${ }^{2}$ Director of Jordan Food and Drug Administration, Jordan University of Science and Technology, Ar-Ramtha, Jordan. \\ ${ }^{3}$ Qatar University, Doha, Qatar.
}

\begin{tabular}{l}
\hline ARTICLE INFO \\
\hline Received on: $11 / 03 / 2021$ \\
Accepted on: $01 / 05 / 2021$ \\
Available online: $05 / 07 / 2021$
\end{tabular}

\section{Key words:}

Skin care, hair care, natural cosmetics, randomized controlled trials.

\begin{abstract}
Cosmetics are marketed and used worldwide for various purposes. Several natural products are used for the development of cosmetic preparations. This paper systematically reviews randomized controlled trials (RCTs) investigating plant extracts, herbal preparations, and isolated plant-derived compounds used particularly for skin and hair care. Two independent electronic searches were conducted through PubMed and EMBASE to identify eligible RCTs. The Preferred Reporting Items for Systematic Reviews and Meta-Analyses statement was followed. Data extraction was performed independently by four authors based on standardized extraction forms. The risk of bias was assessed using the Cochrane Collaboration's tool for assessing the risk of bias in randomized trials. Sixty-three RCTs were identified; 53 were using natural products for skin care and 10 for hair care. The results were summarized in tables including the population, type of intervention, comparisons with placebo or other natural products, outcomes reported, follow-up period (P: Patient, Population; I: Intervention; C: Comparison (or Control); O: Outcome; T: Time), and country in which the study was conducted. Ten plants were identified to be present in different locations in Jordan by referring to the Royal Botanic Gardens' publication, titled "The Plants of Jordan: An Annotated Checklist." Some plants were found to have promising findings requiring further investigations in bigger RCTs with robust design and adequate reporting.
\end{abstract}

\section{INTRODUCTION}

In the highly visual consumer culture, the appearance of body shape and beauty is gaining more attention from people as it has become an important factor in the individual's sense of identity. The human body is the most visible expression of a person's self (Domzal and Kernan, 1993; Shilling, 2017), and as a result, people have a high desire to be physically attractive (Kim and Seock, 2009). One way to do that is by using cosmetic products.

Cosmetics are globally used to enhance the appearance or body odor (Ashawat et al., 2009; Shivanand et al., 2010). Cosmetic products are developed in various dosage forms using natural and synthetic ingredients (Ashawat et al., 2009). The use of

\footnotetext{
*Corresponding Author

Samar Thiab, Faculty of Pharmacy, Applied Science Private University, College of Pharmacy, Qatar University, Amman, Jordan.

E-mail:S_Thiab@asu.edu.jo
}

plants and herbs in cosmetics has gained more popularity in recent years (Ashawat et al., 2009; Gediya et al., 2011; Shivanand et al., 2010). The number of products containing natural ingredients is increasing (Antignac et al., 2011). These products are commonly used with the misconception that they are always more effective and safer than completely synthetic products (Antignac et al., 2011; Ashawat et al., 2009).

Several plants and herbs have the potential for the development of cosmetic preparations due to their chemical composition containing compounds like vitamins, minerals, flavonoids, tannins, and amino acids, which have the potential to influence the human body (Fonseca-Santos et al., 2015; Yoo et al., 2018).

The use of plants and herbs to enhance beauty is well known in the Middle East since around 3000 BC where it was commonly used by ancient Egyptians and Babylonians in Iraq (Sawicka and Noaema, 2015). In the Middle Eastern region, particularly in Jordan, a wide range of plants with medicinal activity are available (Aburjai et al., 2007; Afifi and Abu-Irmaileh, 
2000; Alzweiri et al., 2011). The use of natural ingredients in cosmetics is gaining more popularity, not only for their health benefits but also due to the higher demand by consumers for ecologically friendly products (Laroche et al., 2001; Ribeiro et al., 2015).

This study systematically reviewed randomized controlled trials (RCTs) investigating plant extracts, herbal preparations, and isolated plant-derived compounds used for cosmetic purposes focusing on skin and hair care. In addition, it provides a list of the plants/herbs available in Jordan that have been studied in the literature for cosmetic purposes by referring to the Royal Botanic Gardens publication titled "The Plants of Jordan: An Annotated Checklist”' (Taifour et al., 2017).

\section{MATERIALS AND METHODS}

This systematic review (SR) was based on a registered (PROSPERO CRD42020198926) protocol and reported in line with the Preferred Reporting Items for Systematic Reviews and Meta-Analyses (PRISMA) statement (Moher et al., 2009).

\section{Data sources and searches}

RCTs reporting cosmetic clinical outcomes in adults ( $\geq 18$ years old) in the English language were included. Exclusion criteria included studies involving trials with interventions requiring medical attention and postprocedural treatment, not listing a clear description of botanical/phytochemical intervention.

Eligible trials were identified by electronic searches in PubMed and EMBASE from the beginning of time on the database until 26/7/2020. A combination of the following medical subject heading (MeSH terms) and free-text terms was used: phytotherapy, herbal medicine, plant extract, volatile oil, cosmetics, and skin care.

Study selection

Two authors independently reviewed the trial inclusion and exclusion criteria. Excluded trials were listed with the reason for exclusion (Supplementary Material). Disagreements were resolved by consulting a third researcher and achieving consensus.

\section{Data extraction and quality assessment}

Data extraction was performed independently by four authors based on standardized extraction forms. Each article was independently extracted by two authors. Disagreements were resolved through discussions and the corresponding authors were contacted if any of the pieces of required information were not described in the published manuscripts.

The risk of bias was assessed by separate domains: randomization, allocation concealment, selective reporting, blinding of participants and authors, attrition, and other biases using the Cochrane Collaboration's tool for assessing the risk of bias in randomized trials (Higgins et al., 2011). The results of these domains were graded as a "low," "high," or "unclear" risk of bias.

\section{RESULTS}

\section{Skin care}

The initial search yielded 1,987 abstracts. Removal of duplicates and applying the exclusion criteria identified 63 studies,
53 of which employed natural products for skin care and 10 for hair care. The process of selecting the studies included in this literature review was based on the inclusion and exclusion criteria as illustrated in Figure 1.

The 63 included studies are summarized in Tables 1 and 2 to demonstrate plants, herbs, or isolated compounds tested in the selected RCTs for skin care and hair care, respectively.

The main skin conditions addressed in selected RCTs were acne, hyperpigmentation, wrinkles, hirsutism, inflammation, stretch marks, and scars as well as testing plants and herbs for their moisturizing and skin protection properties. For hair care, the main hair issues addressed in the RCTs were hair thinning and dandruff.

The highest number of RCTs concerning selected skin conditions tested natural products for their skin protection properties $(n=12)$ and was published between 1997 and 2018. The investigated plants/products included Polypodium leucotomos (Gonzalez et al., 1997), Camellia sinensis (Camouse et al., 2009), Hibiscus abelmoschus (Rival et al., 2009), Calendula officinalis (Akhtar et al., 2011), Avena sativa (Michelle, 2016), Ribes nigrum (Ray et al., 2016), phenolic veratric acid (Lee et al., 2016), and Cucumis melo (Egoumenides et al., 2018), as a single ingredient within the formulation. Four other RCTs investigated the combination of extracts, including soy and jasmine (Bazin et al., 2010), dead sea water and Himalayan extracts (Wineman et al., 2012), Olea europaea and Helianthus annuus (Danby et al., 2013), and Portulaca oleracea and Prinsepia utilis (Wang et al., 2018).

The second highest number of selected RCTs addressed antiaging effects $(n=10)$ and was published between 2000 and 2020. The plants/herbs investigated included Centella asiatica (Gonzalez et al., 1997), date palm kernel (Bauza et al., 2002), Sanguisorba officinalis (Kim et al., 2008), Platycarya strobilacea (Kim et al., 2010), Oryza sativa (Kanlayavattanakul et al., 2016), Geranium thunbergii (Yoshida et al., 2019), Psoralea corylifolia (Goldberg et al., 2019), and Zanthoxylum bungeanum (Zeng et al., 2019) and two used a combination of extracts including Glycyrrhiza glabra, Angelica gigas, Prunus persica, Ophiopogon japonicus, Paeonia suffruticosa, Atractylodes japonica, Poria cocos, Rehmannia chinensis, Cimicifuga simplex, Asparagus cochinchinensis, Scutellaria baicalensis, Astragalus membranaceus, Carthamus tinctorius (Roh et al., 2019), and Coptis teeta with Trichosanthes rosthornii (Im et al., 2020).

Nine studies published between 2012 and 2020 tested natural products for the treatment of hyperpigmentation. These studies investigated the constituents Silybum marianum (Altaei, 2012), Sophora flavescens (Shin et al., 2013), Polypodium leucotomos (White et al., 2013), Rumex occidentalis (Mendoza et al., 2014), Serratula quinquefolia (Morag et al., 2015), P. cocos Wolf (Lee and Cha, 2018), Vitis vinifera (Tsuchiya et al., 2020), and O. europaea (de Toledo Bagatin et al., 2020); one study used a combination of China camellia, Sanchi, P. utilis, and P. oleracea (Zhang et al., 2019).

Next were RCTs investigating natural products, examining their ability to reduce body hair growth $(n=5)$; these studies were published between 2003 and 2019 and included Foeniculum vulgare (Javidnia et al., 2003), Stryphnodendron 


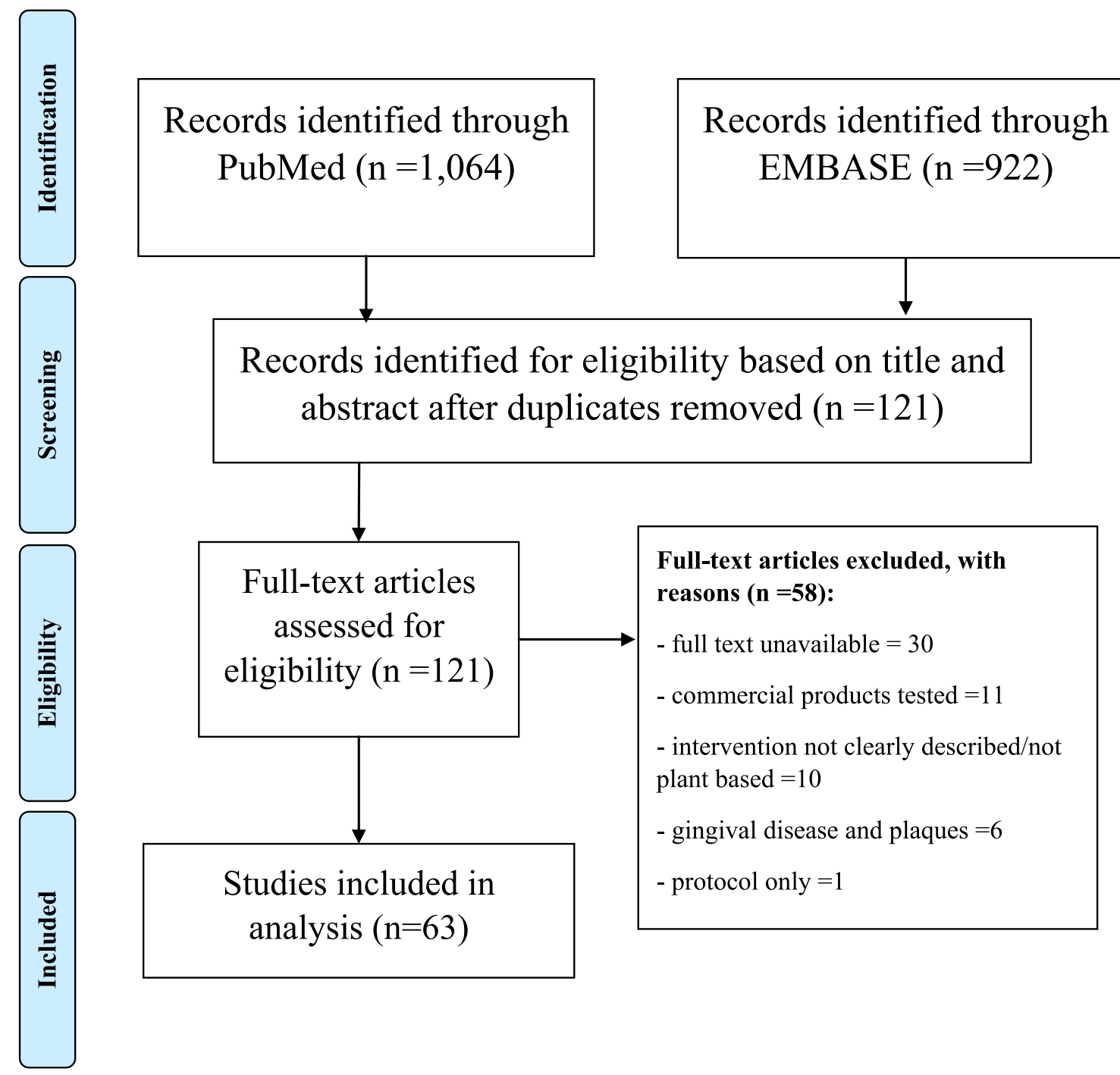

Figure 1. PRISMA flow diagram showing the number of RCTs identified and included in this SR.

adstringens (Vicente et al., 2009), Medicago sativa (Aali et al., 2016), and Curcuma aeruginosa (Srivilai et al., 2017, 2018).

Studies concerning the moisturizing properties of natural products $(n=4)$ were published between 2016 and 2019 and included Rhododendron ferrugineum (Filipovic et al., 2016), C. asiatica (Milani and Sparavigna, 2017), Scaphium scaphigerum (Kanlayavattanakul et al., 2017), and Curcuma longa (Asada et al., 2019).

The RCTs investigating natural products for their antiinflammatory properties $(n=3)$ were published between 2014 and 2019, investigating the constituents Prunus yedoensis (Zhang et al., 2014) and Glycyrrhiza inflata (Boonchai et al., 2018) as a single ingredient preparation within the formulation; one related RCT investigated a combination of extracts of Gentiana lutea, $G$. glabra, and Salix daphnoides (Seiwerth et al., 2019).

The RCTs investigating natural products for acne treatment $(n=2)$ were published between 2011 and 2018 and explored combinations of extracts; the first group of studies explored retinol, rose, and hexamidine diisethionate (Lee et al., 2011), while the second group explored coco-glucoside,
Simmondsia chinensis, G. lutea, Mentha arvensis, Humulus lupulus, Leptospermum scoparium, S. daphnoides, H. annuus, pectin, and xanthan gum (Weber et al., 2019).

Two studies, published between 2008 and 2016, tested natural products for foot care. In the former, they used a combination of mango butter and olein fraction fortified with vitamin E (Mandawgade and Patravale, 2008), whereas in the latter study, Ziziphus mauritiana (Akhtar et al., 2016) was used.

Rosacea was investigated in one study published in 2015 and used a cream containing medical-grade kanuka honey (Braithwaite et al., 2015). Stretch marks and scars reduction were investigated in two separate studies published in 2014 and 2010, using O. europaea (Soltanipour et al., 2014) and Allium cepa (Hosnuter et al., 2007), respectively.

Finally, three studies published in 2015, 2018, and 2019 tested plants for multiple effects; the first study investigated Tamarindus indica for its antisebum and antihyperpigmentation properties (Muhammad et al., 2015); the second tested Prunus serrulata for its moisturizing, antihyperpigmentation, antiaging, and overall improvement of skin condition and elasticity 


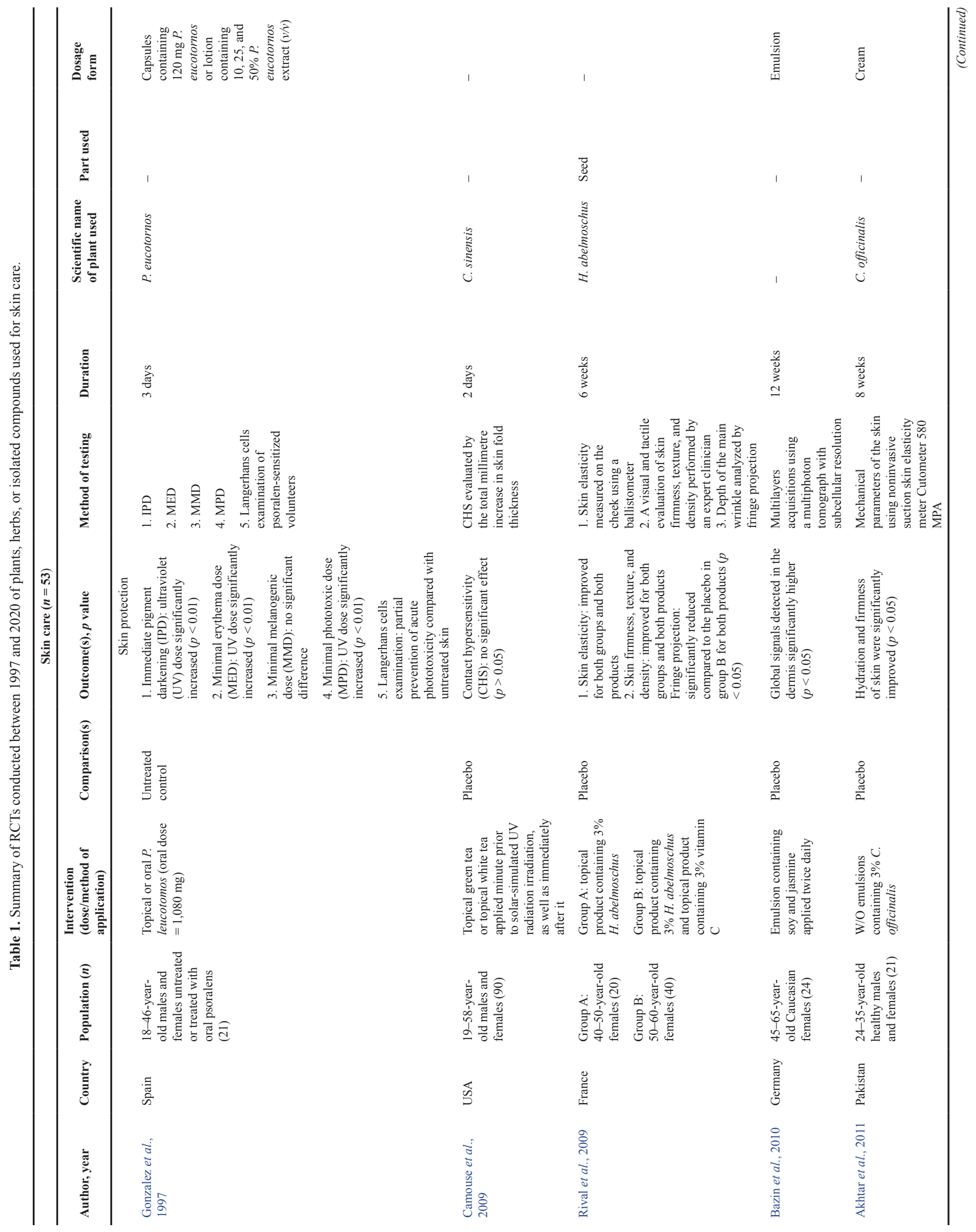




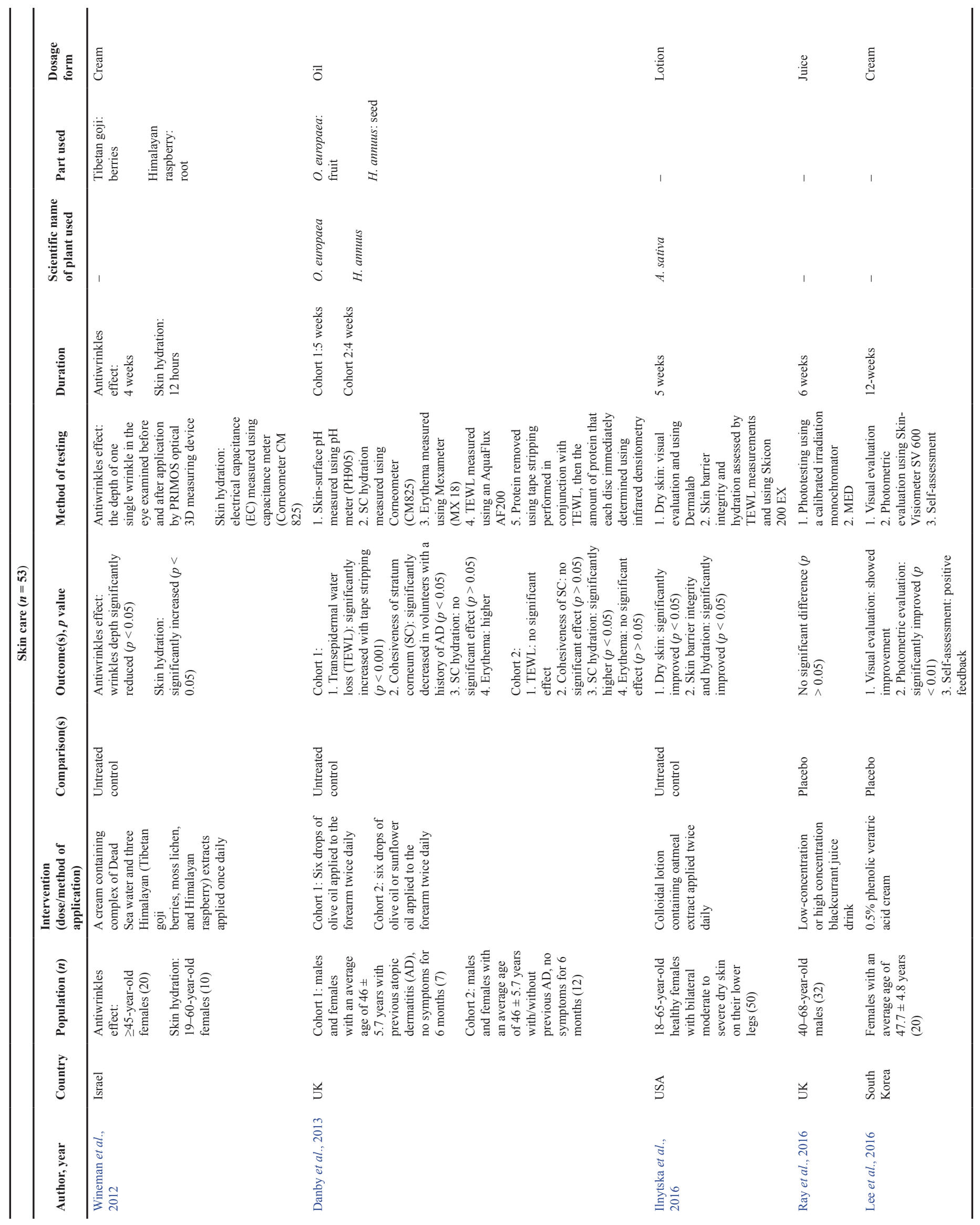




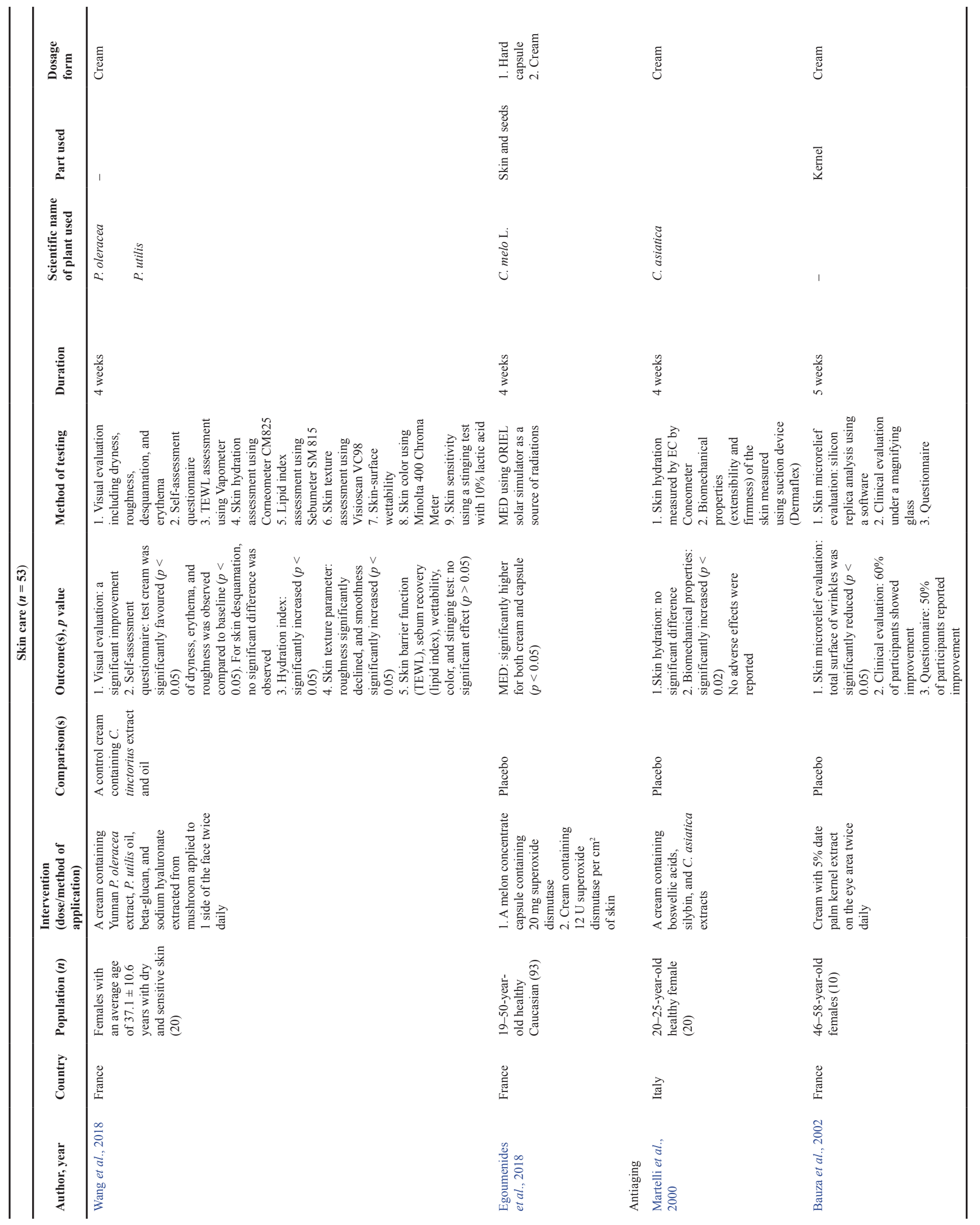




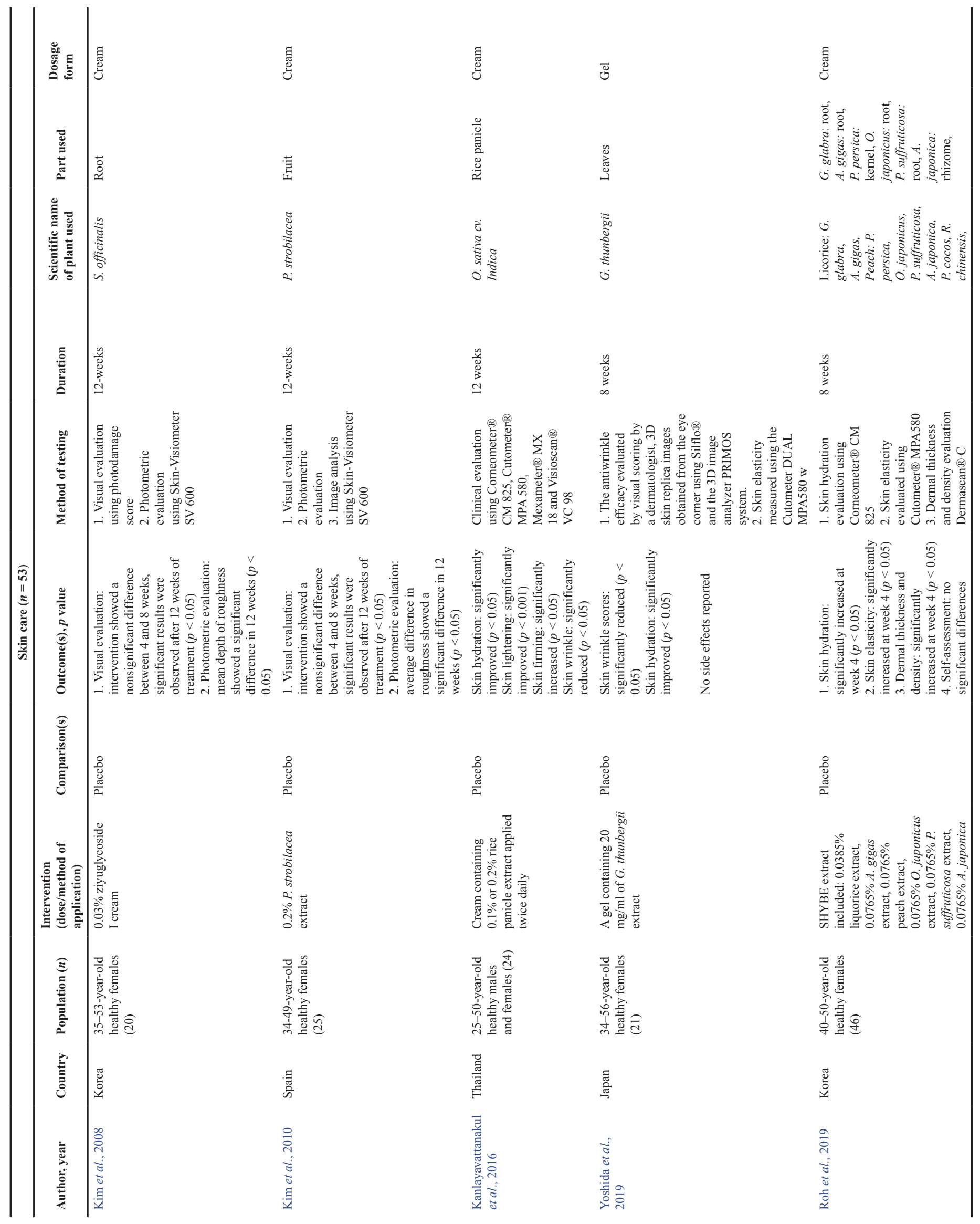




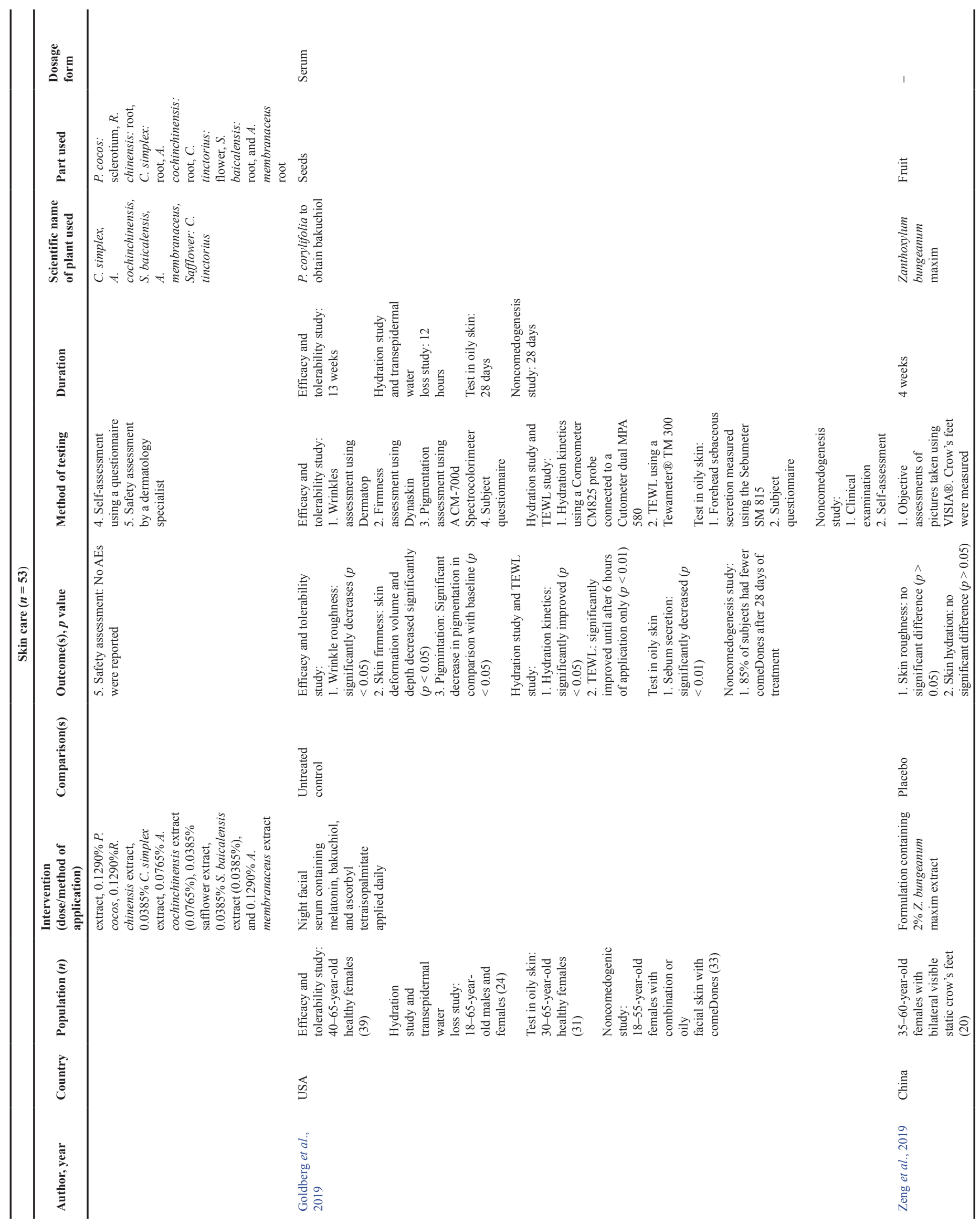




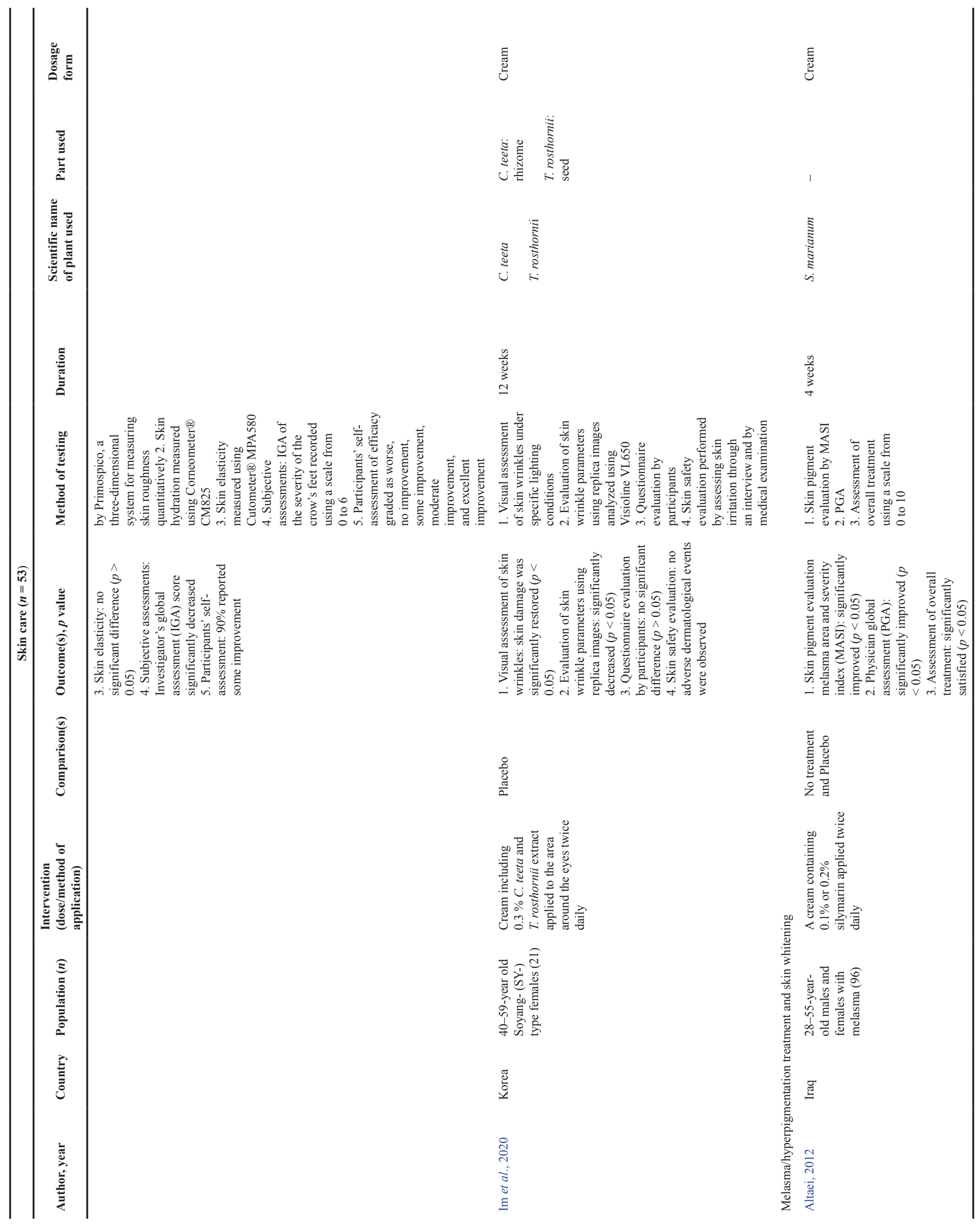




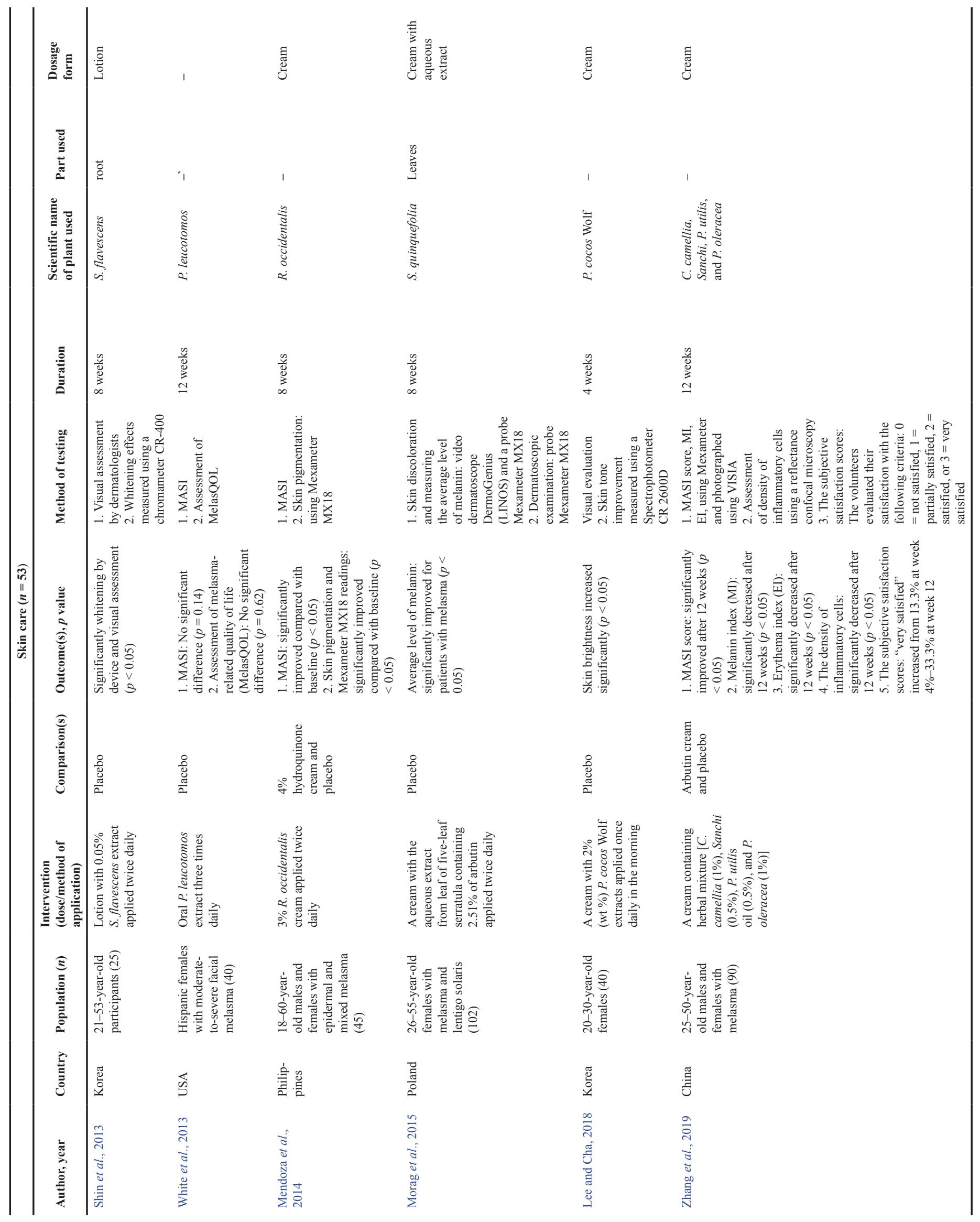




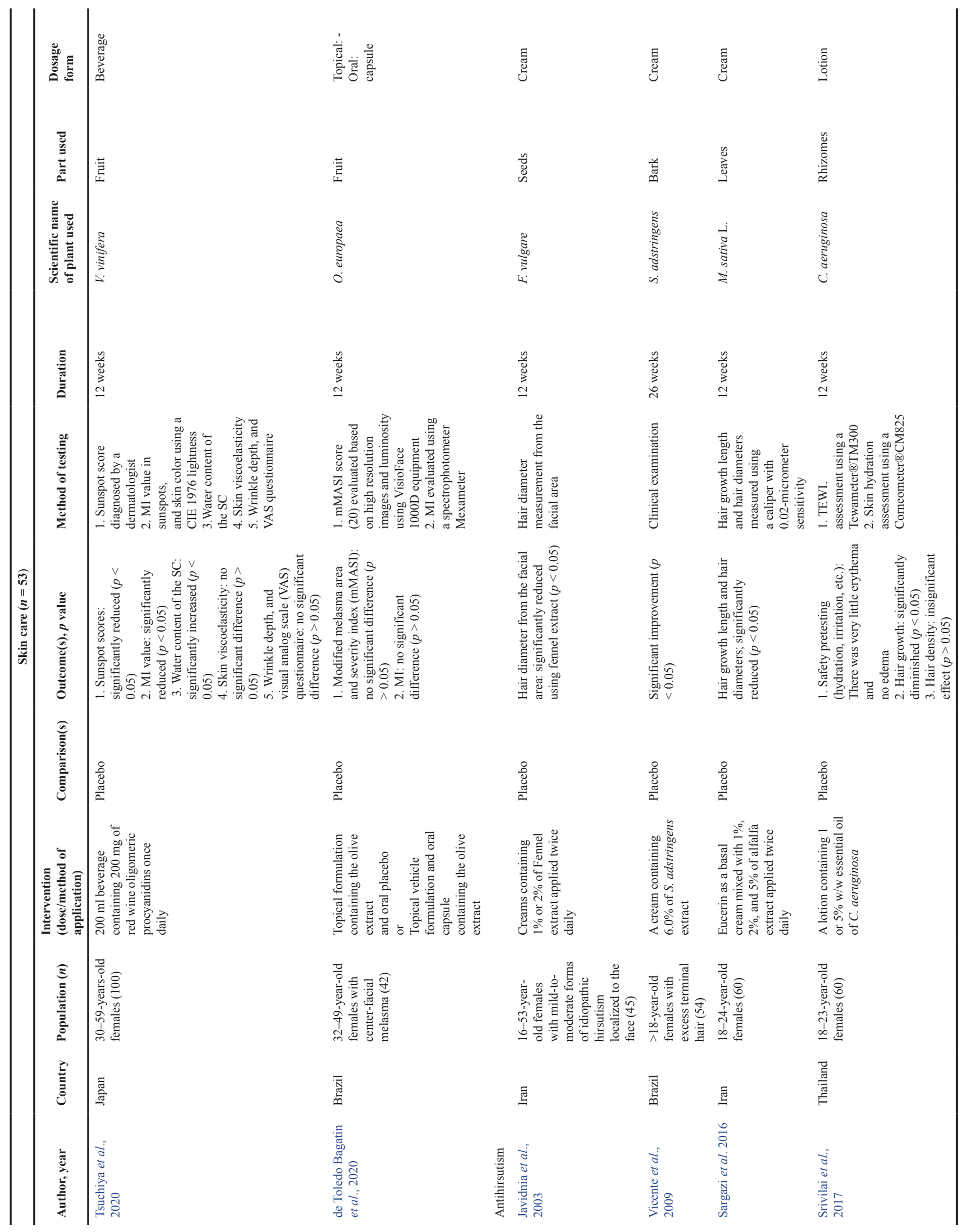




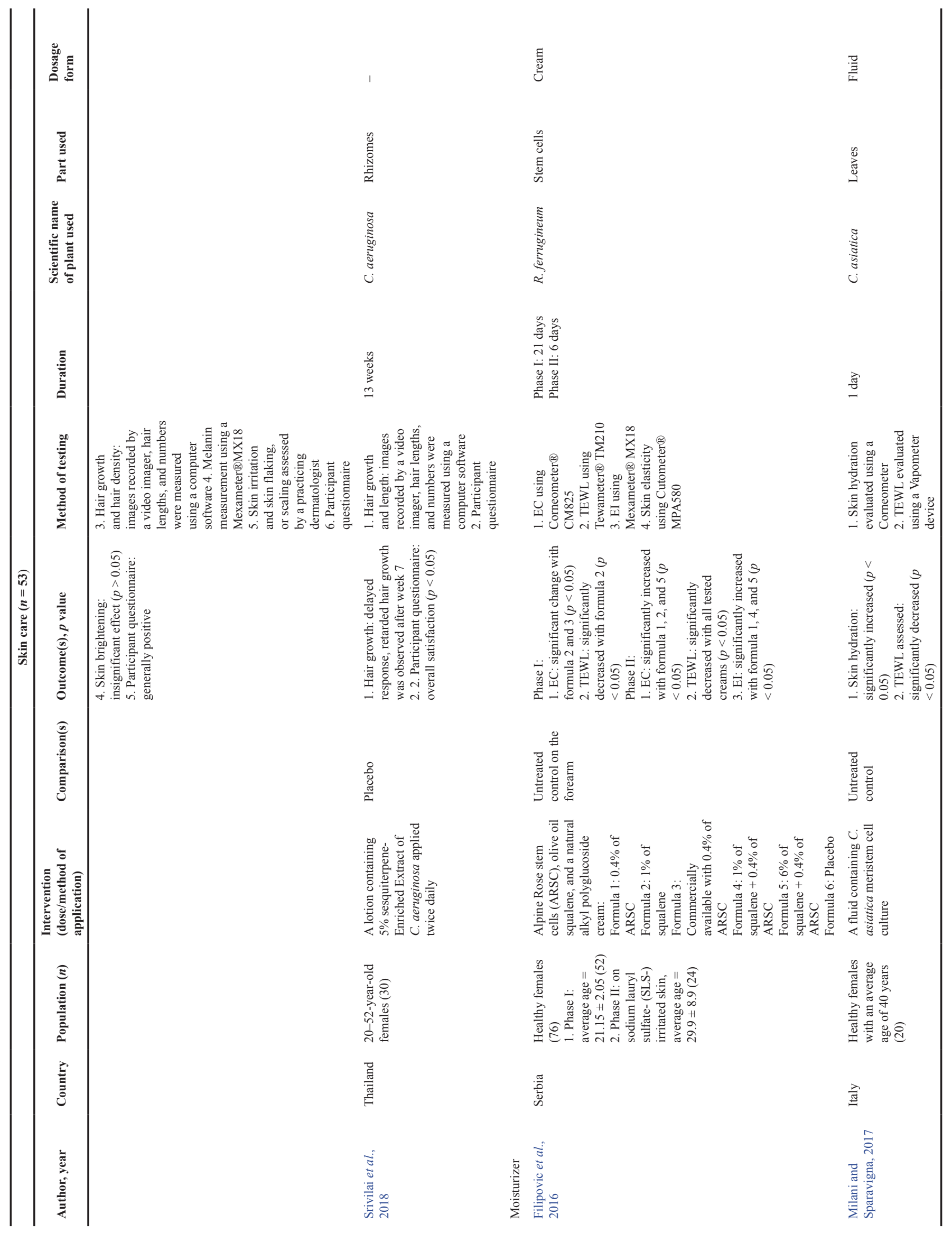




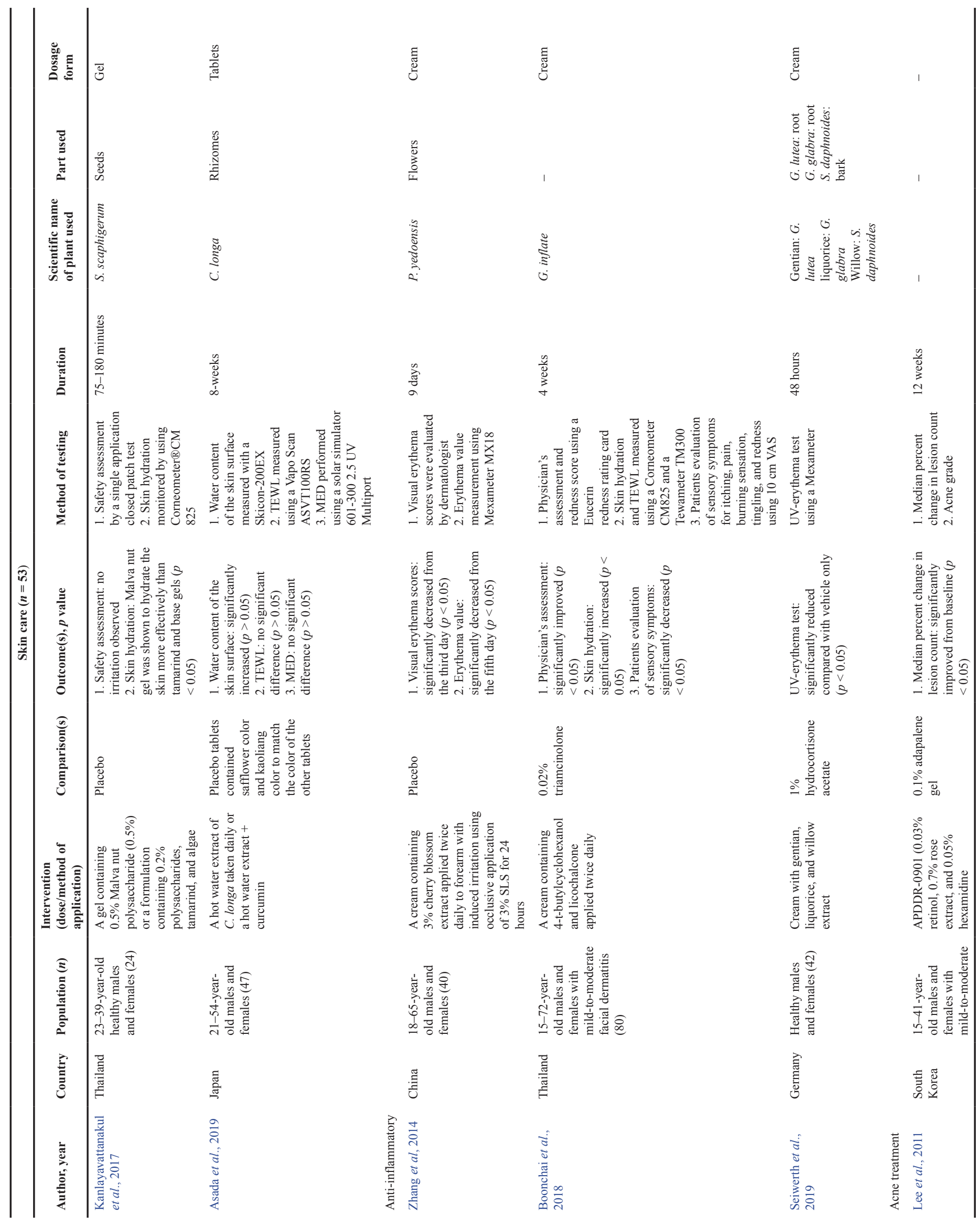




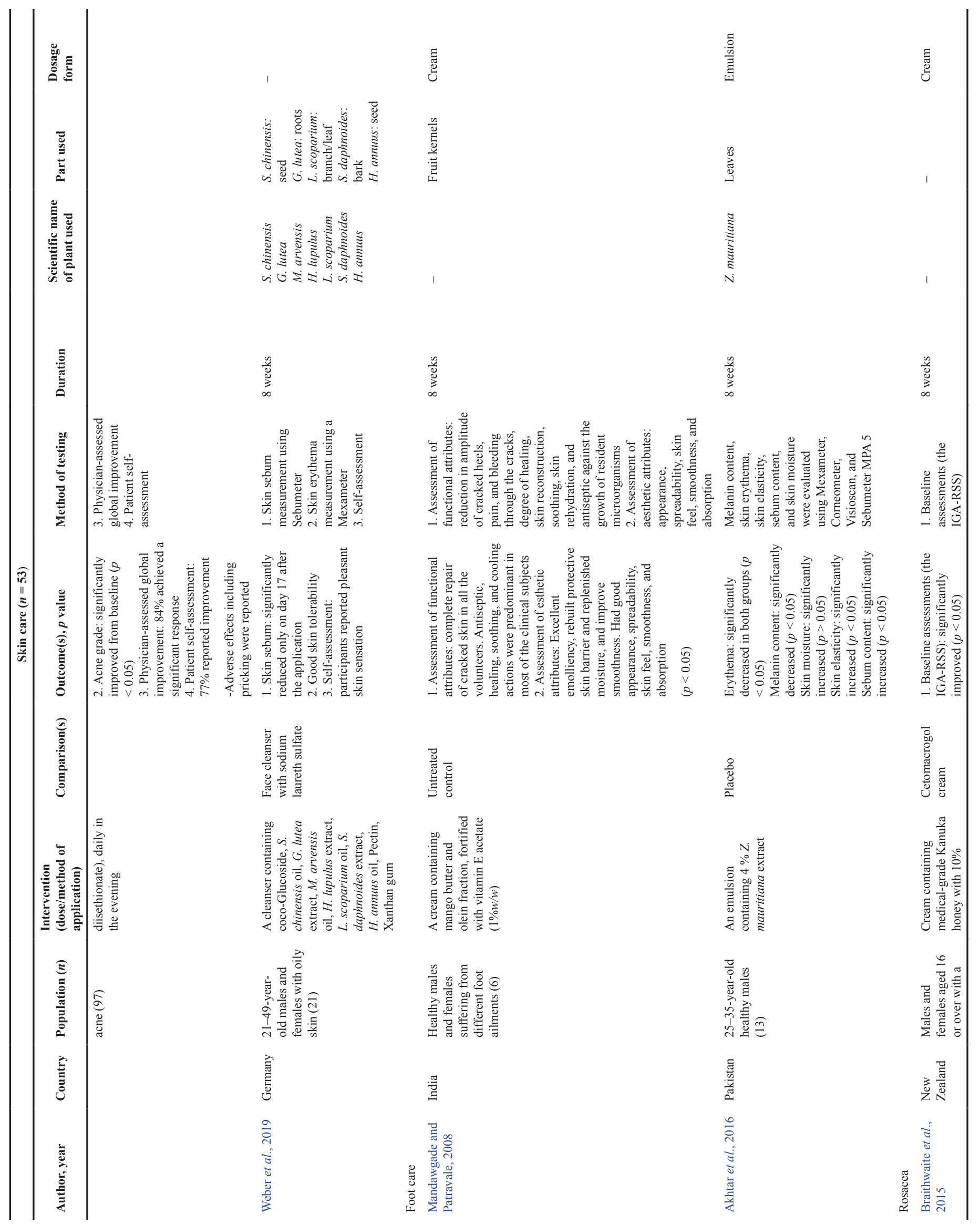




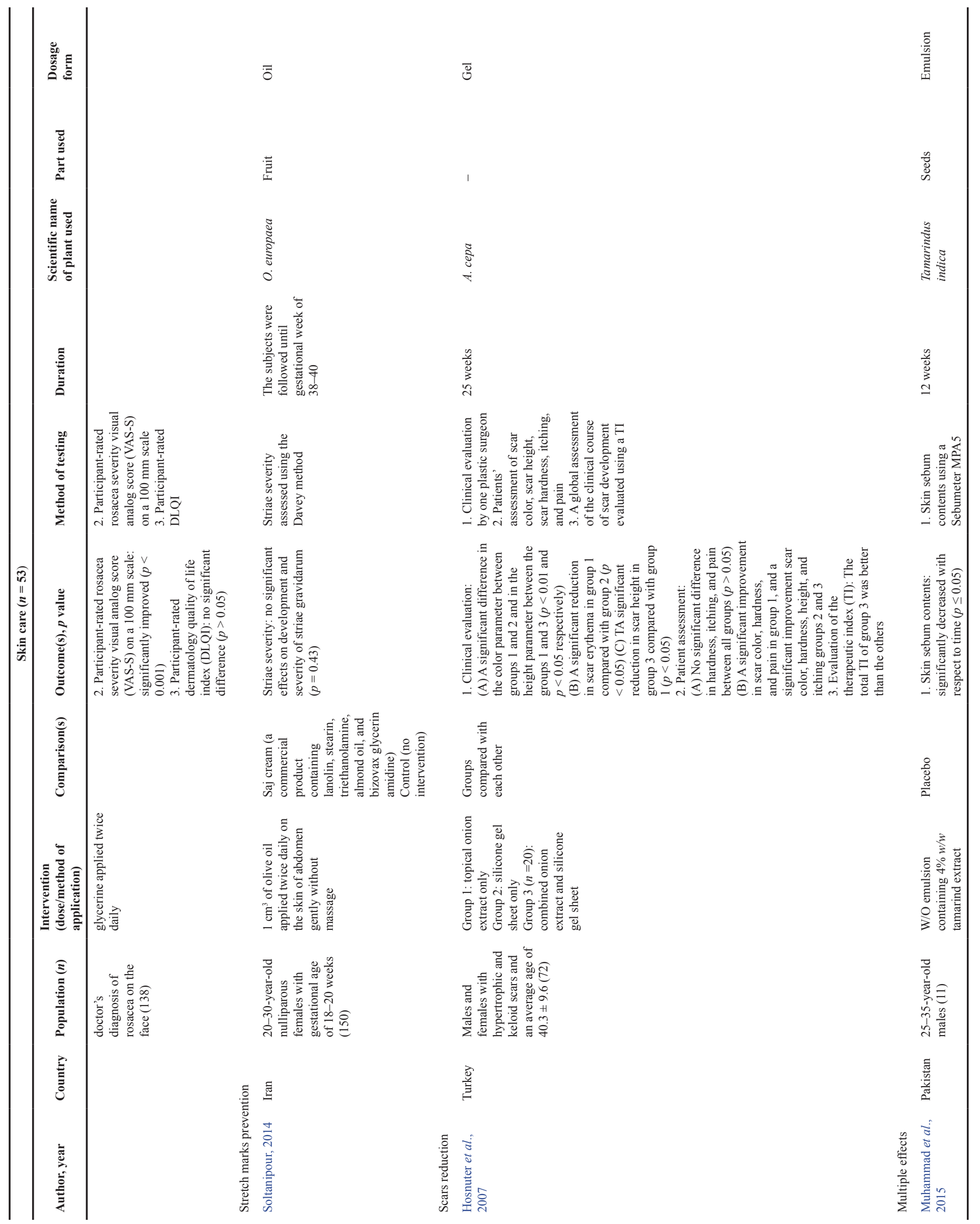




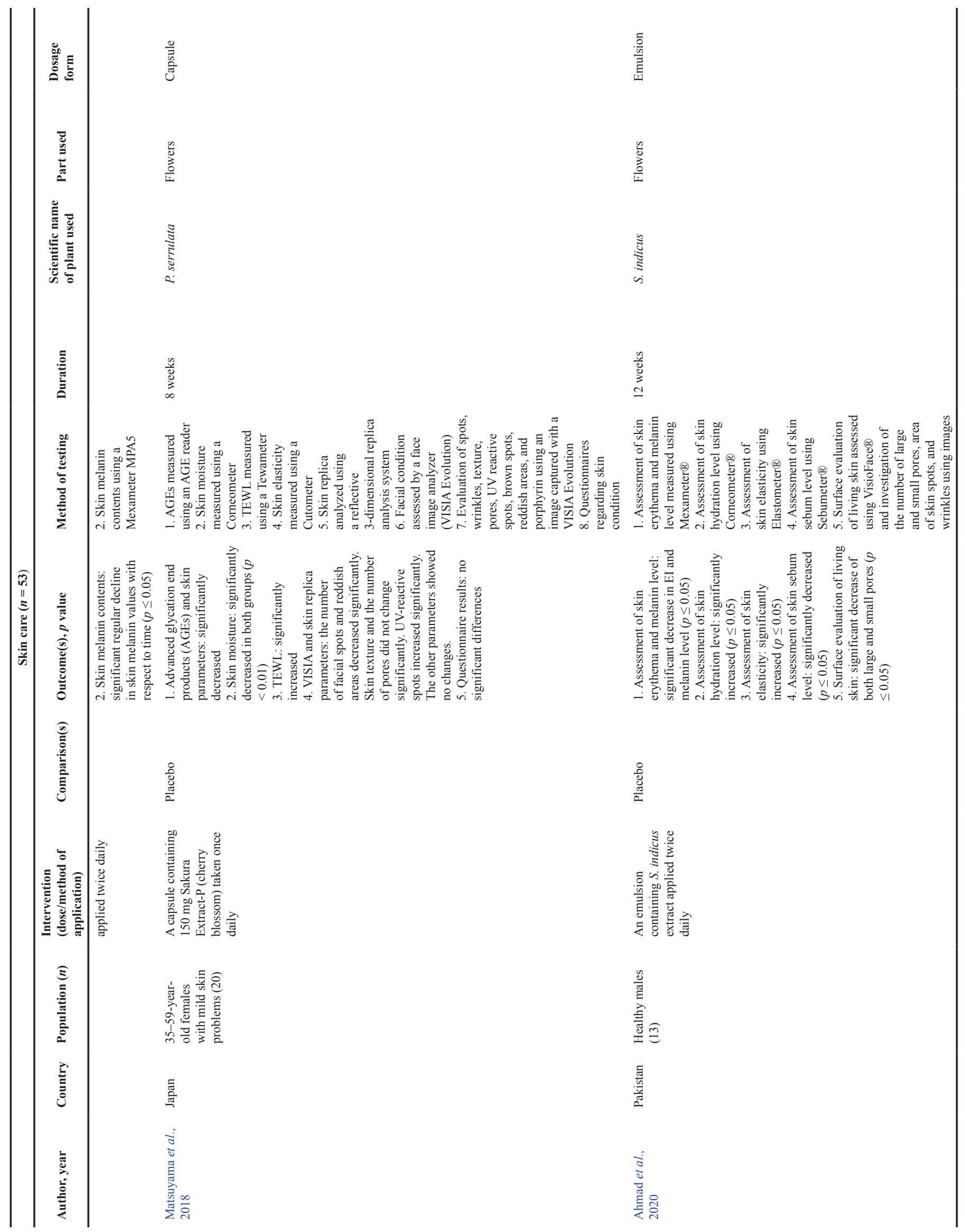




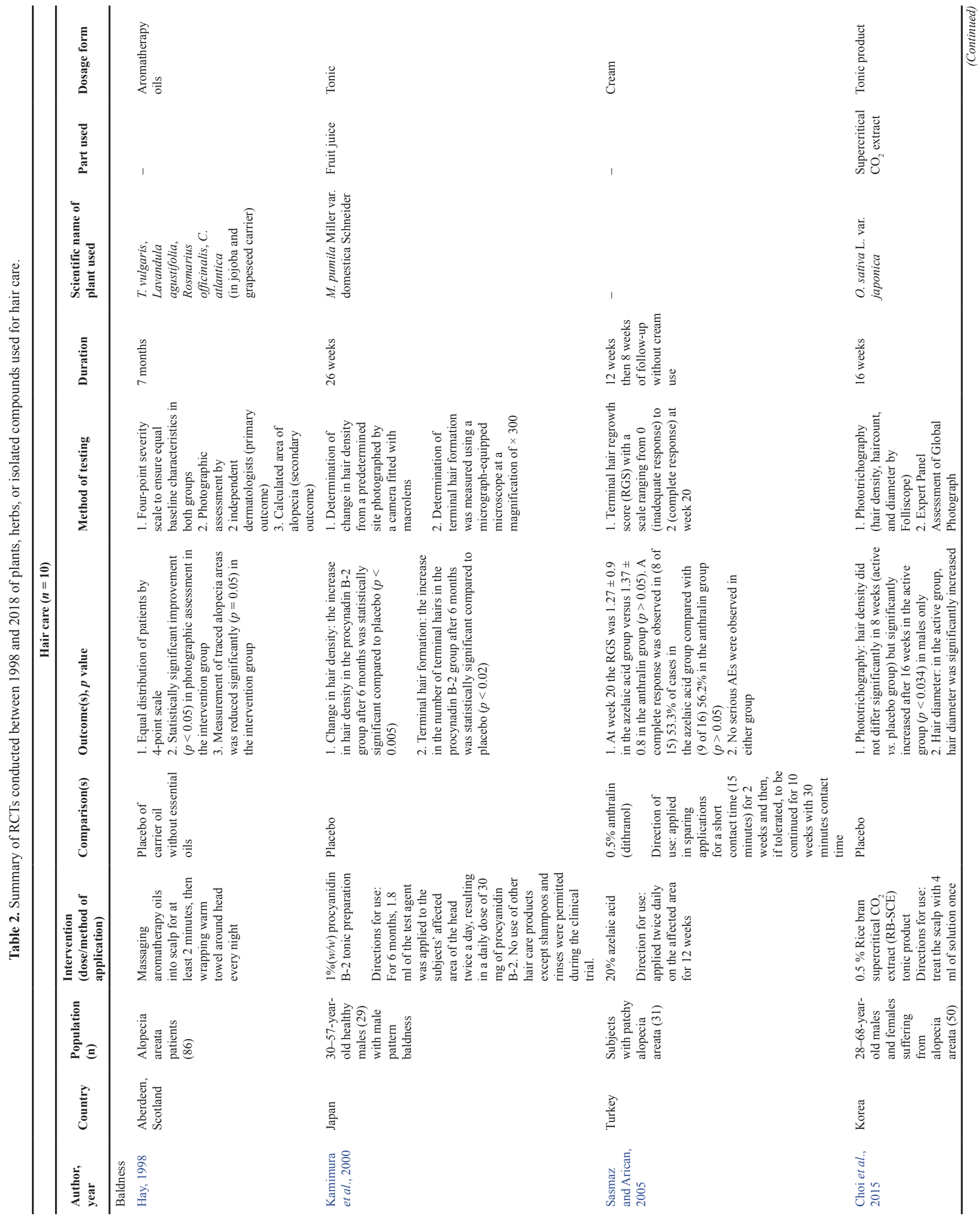




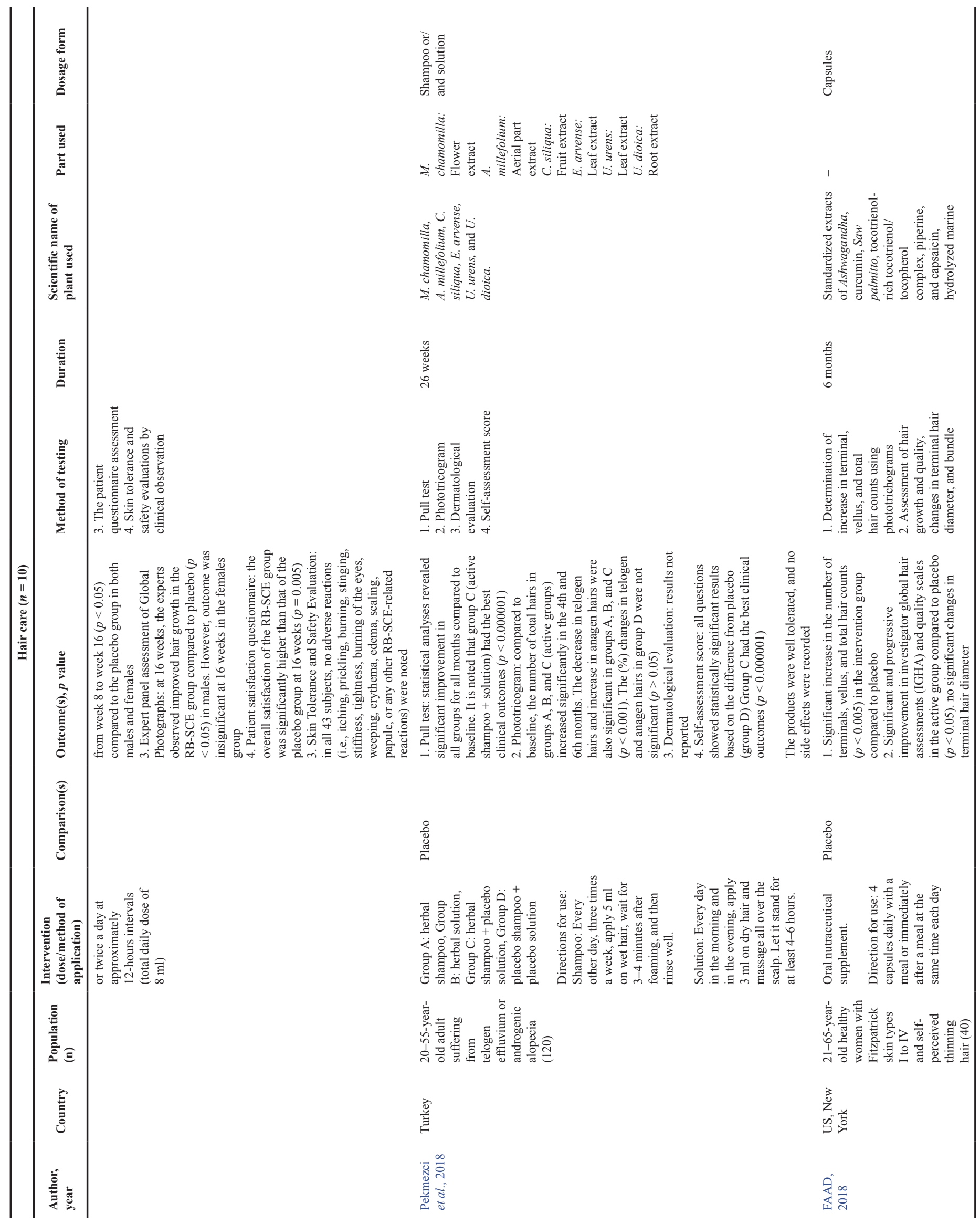




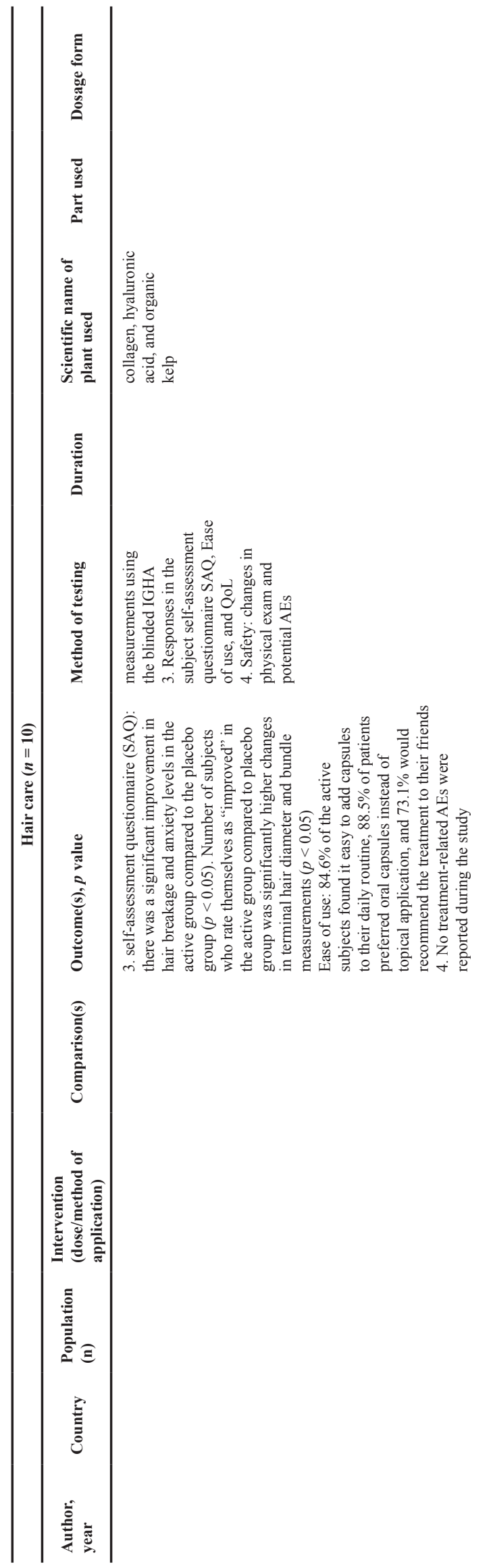

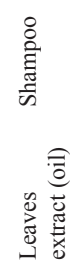

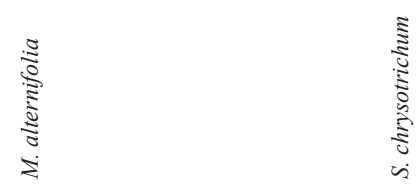

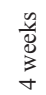
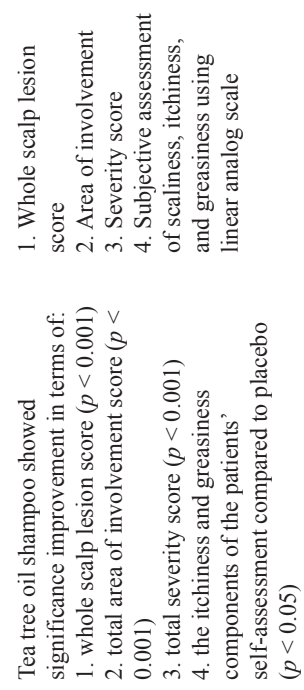

$\frac{8}{\stackrel{\Xi}{2}}$
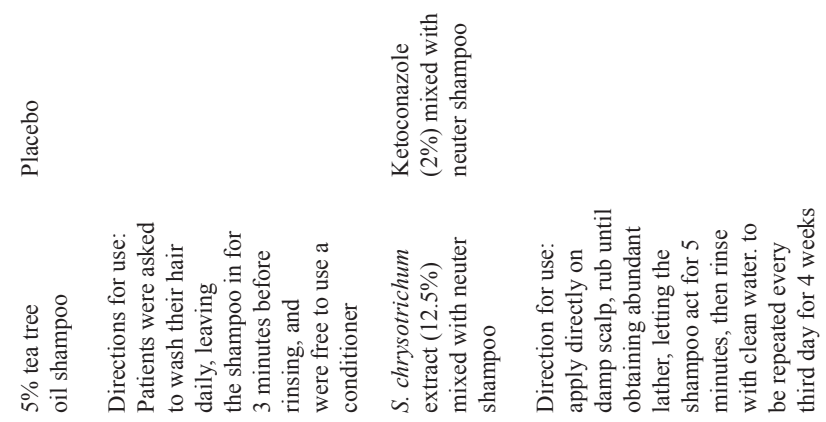

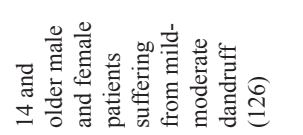
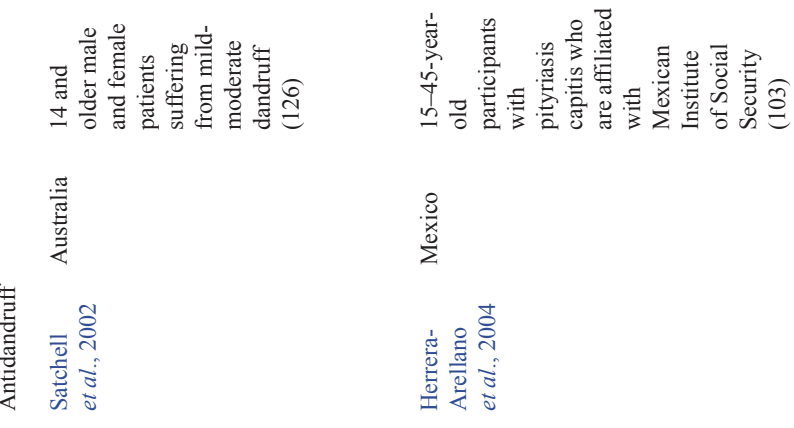

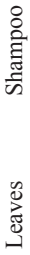

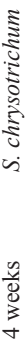
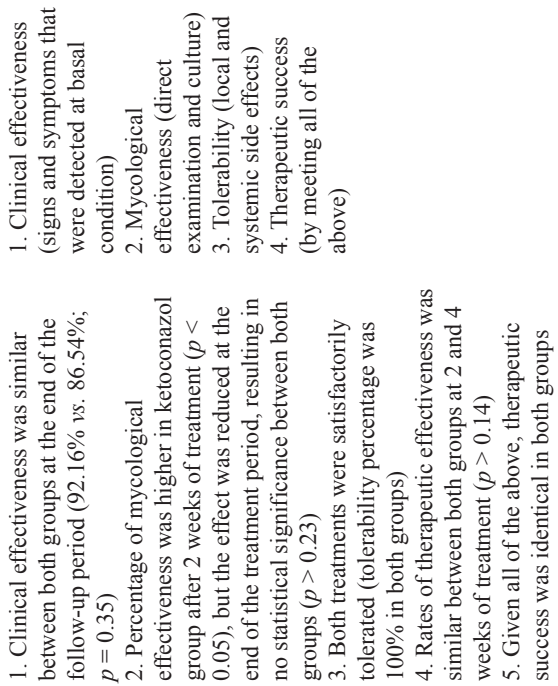

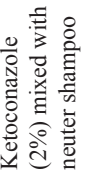

$\stackrel{8}{\stackrel{8}{*}}$

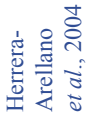


Thiab et al. / Journal of Applied Pharmaceutical Science 11 (07); 2021: 020-045

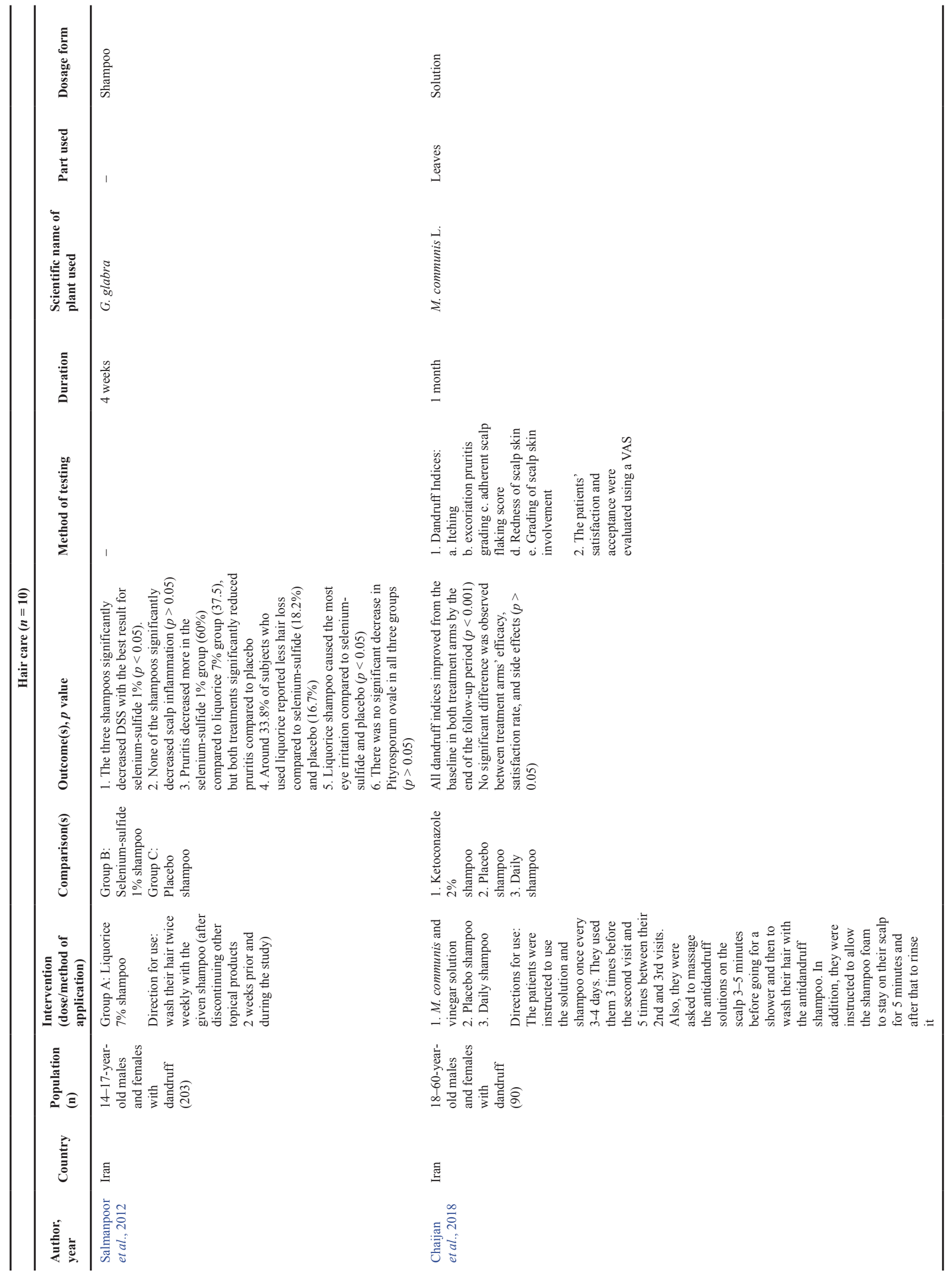


Thiab et al. / Journal of Applied Pharmaceutical Science 11 (07); 2021: 020-045

Table 3. Risk of bias assessment of the RCTs included in this SR.

\begin{tabular}{|c|c|c|c|c|c|c|c|}
\hline \multicolumn{2}{|c|}{ Selection bias } & \multirow{2}{*}{$\begin{array}{c}\text { Reporting bias } \\
\begin{array}{c}\text { Selective } \\
\text { reporting }\end{array}\end{array}$} & \multirow{2}{*}{$\begin{array}{c}\text { Other biases } \\
\begin{array}{c}\text { Other sources } \\
\text { of bias }\end{array}\end{array}$} & \multirow{2}{*}{$\begin{array}{c}\text { Performance bias } \\
\text { Blinding } \\
\text { (participants and } \\
\text { personnel) }\end{array}$} & \multirow{2}{*}{$\begin{array}{c}\text { Detection bias } \\
\text { Blinding } \\
\text { (outcome } \\
\text { assessment) }\end{array}$} & \multirow{2}{*}{$\begin{array}{c}\text { Attrition bias } \\
\begin{array}{c}\text { Incomplete } \\
\text { outcome data }\end{array}\end{array}$} & \\
\hline $\begin{array}{c}\text { Random sequence } \\
\text { generation }\end{array}$ & $\begin{array}{c}\text { Allocation } \\
\text { concealment }\end{array}$ & & & & & & \\
\hline & & & kin care $\mathrm{RCTs}$ & & & & \\
\hline $\mathrm{X}$ & $\mathrm{X}$ & $\mathrm{L}$ & $\mathrm{H}$ & $\mathrm{X}$ & $\mathrm{X}$ & $\mathrm{L}$ & Gonzalez et al., 1997 \\
\hline $\mathrm{X}$ & $\mathrm{X}$ & $\mathrm{L}$ & $\mathrm{L}$ & $\mathrm{L}$ & $\mathrm{L}$ & $\mathrm{X}$ & Martelli et al., 2000 \\
\hline $\mathrm{L}$ & $\mathrm{X}$ & $\mathrm{L}$ & $\mathrm{L}$ & $\mathrm{L}$ & $\mathrm{L}$ & $\mathrm{L}$ & Bauza et al., 2002 \\
\hline $\mathrm{L}$ & $\mathrm{X}$ & $\mathrm{L}$ & $\mathrm{L}$ & $\mathrm{L}$ & $\mathrm{L}$ & $\mathrm{L}$ & Javidnia et al., 2003 \\
\hline $\mathrm{L}$ & $\mathrm{X}$ & $\mathrm{L}$ & $\mathrm{L}$ & $\mathrm{L}$ & $\mathrm{L}$ & $\mathrm{L}$ & Javidnia et al., 2003 \\
\hline $\mathrm{H}$ & $\mathrm{X}$ & $\mathrm{L}$ & $\mathrm{L}$ & $\mathrm{X}$ & $\mathrm{H}$ & $\mathrm{L}$ & Hosnuter et al., 2007 \\
\hline $\mathrm{X}$ & $\mathrm{X}$ & $\mathrm{L}$ & $\mathrm{L}$ & $\mathrm{L}$ & $\mathrm{L}$ & $\mathrm{L}$ & Camouse et al., 2009 \\
\hline $\mathrm{X}$ & $\mathrm{X}$ & $\mathrm{L}$ & $\mathrm{L}$ & $\mathrm{L}$ & $\mathrm{L}$ & $\mathrm{L}$ & Kim et al., 2008 \\
\hline $\mathrm{X}$ & $\mathrm{X}$ & $\mathrm{L}$ & $\mathrm{X}$ & $\mathrm{X}$ & $\mathrm{X}$ & $\mathrm{X}$ & Mandawgade and Patravale, 2008 \\
\hline $\mathrm{L}$ & $\mathrm{X}$ & $\mathrm{L}$ & $\mathrm{L}$ & $\mathrm{L}$ & $\mathrm{L}$ & $\mathrm{X}$ & Rival et al., 2009 \\
\hline $\mathrm{X}$ & $\mathrm{L}$ & $\mathrm{L}$ & $\mathrm{L}$ & $\mathrm{L}$ & $\mathrm{L}$ & $\mathrm{H}$ & Vicente et al., 2009 \\
\hline $\mathrm{L}$ & $\mathrm{X}$ & $\mathrm{L}$ & $\mathrm{L}$ & $\mathrm{L}$ & $\mathrm{X}$ & $\mathrm{L}$ & Bazin et al., 2010 \\
\hline $\mathrm{X}$ & $\mathrm{X}$ & $\mathrm{L}$ & $\mathrm{L}$ & $\mathrm{L}$ & $\mathrm{L}$ & $\mathrm{L}$ & Kim et al., 2010 \\
\hline $\mathrm{X}$ & $\mathrm{X}$ & $\mathrm{L}$ & $\mathrm{L}$ & $\mathrm{L}$ & $\mathrm{H}$ & $\mathrm{X}$ & Akhtar et al., 2011 \\
\hline $\mathrm{L}$ & $\mathrm{L}$ & $\mathrm{L}$ & $\mathrm{L}$ & $\mathrm{L}$ & $\mathrm{L}$ & $\mathrm{H}$ & Lee et al., 2011 \\
\hline $\mathrm{X}$ & $\mathrm{X}$ & $\mathrm{L}$ & $\mathrm{L}$ & $\mathrm{L}$ & $\mathrm{L}$ & $\mathrm{L}$ & Altaei, 2012 \\
\hline $\mathrm{H}$ & $\mathrm{H}$ & $\mathrm{L}$ & $\mathrm{L}$ & $\mathrm{H}$ & $\mathrm{H}$ & $\mathrm{L}$ & Wineman et al., 2012 \\
\hline $\mathrm{X}$ & $\mathrm{X}$ & $\mathrm{L}$ & $\mathrm{L}$ & $\mathrm{H}$ & $\mathrm{L}$ & $\mathrm{L}$ & Danby et al., 2013 \\
\hline $\mathrm{X}$ & $\mathrm{X}$ & $\mathrm{L}$ & $\mathrm{L}$ & $\mathrm{L}$ & $\mathrm{L}$ & $\mathrm{L}$ & Shin et al., 2013 \\
\hline $\mathrm{X}$ & $\mathrm{X}$ & $\mathrm{L}$ & $\mathrm{L}$ & $\mathrm{L}$ & $\mathrm{L}$ & $\mathrm{L}$ & White et al., 2013 \\
\hline $\mathrm{L}$ & $\mathrm{X}$ & $\mathrm{L}$ & $\mathrm{L}$ & $\mathrm{L}$ & $\mathrm{L}$ & $\mathrm{L}$ & Mendoza et al., 2014 \\
\hline $\mathrm{L}$ & $\mathrm{X}$ & $\mathrm{L}$ & $\mathrm{L}$ & $\mathrm{L}$ & $\mathrm{H}$ & $\mathrm{L}$ & Soltanipoura et al., 2014 \\
\hline $\mathrm{X}$ & $\mathrm{X}$ & $\mathrm{L}$ & $\mathrm{L}$ & $\mathrm{H}$ & $\mathrm{L}$ & $\mathrm{L}$ & Zhang et al., 2014 \\
\hline $\mathrm{L}$ & $\mathrm{L}$ & $\mathrm{L}$ & $\mathrm{H}$ & $\mathrm{H}$ & $\mathrm{L}$ & $\mathrm{L}$ & Braithwaite et al., 2015 \\
\hline $\mathrm{H}$ & $\mathrm{X}$ & $\mathrm{L}$ & $\mathrm{L}$ & $\mathrm{L}$ & $\mathrm{L}$ & $\mathrm{L}$ & Morag et al., 2015 \\
\hline $\mathrm{X}$ & $\mathrm{X}$ & $\mathrm{L}$ & $\mathrm{L}$ & $\mathrm{H}$ & $\mathrm{L}$ & $\mathrm{L}$ & Muhammad et al., 2015 \\
\hline $\mathrm{X}$ & $\mathrm{X}$ & $\mathrm{L}$ & $\mathrm{X}$ & $\mathrm{X}$ & $\mathrm{X}$ & $\mathrm{X}$ & Akhtar et al., 2016 \\
\hline $\mathrm{X}$ & $\mathrm{X}$ & $\mathrm{L}$ & $\mathrm{L}$ & $\mathrm{L}$ & $\mathrm{L}$ & $\mathrm{L}$ & Filipovic et al., 2016 \\
\hline $\mathrm{X}$ & $\mathrm{X}$ & $\mathrm{L}$ & $\mathrm{L}$ & $\mathrm{H}$ & $\mathrm{L}$ & $\mathrm{L}$ & Ilnytska et al., 2016 \\
\hline $\mathrm{X}$ & $\mathrm{X}$ & $\mathrm{L}$ & $\mathrm{L}$ & $\mathrm{L}$ & $\mathrm{L}$ & $\mathrm{L}$ & Kanlayavattanakul et al., 2016 \\
\hline $\mathrm{X}$ & $\mathrm{X}$ & $\mathrm{L}$ & $\mathrm{L}$ & $\mathrm{L}$ & $\mathrm{L}$ & $\mathrm{L}$ & Lee et al., 2016 \\
\hline $\mathrm{L}$ & $\mathrm{L}$ & $\mathrm{L}$ & $\mathrm{L}$ & $\mathrm{L}$ & $\mathrm{L}$ & $\mathrm{L}$ & Ray et al., 2016 \\
\hline $\mathrm{L}$ & $\mathrm{L}$ & $\mathrm{L}$ & $\mathrm{L}$ & $\mathrm{L}$ & $\mathrm{L}$ & $\mathrm{L}$ & Srivilai et al., 2017 \\
\hline $\mathrm{L}$ & $\mathrm{X}$ & $\mathrm{L}$ & $\mathrm{L}$ & $\mathrm{H}$ & $\mathrm{L}$ & $\mathrm{L}$ & Boonchai et al., 2018 \\
\hline $\mathrm{X}$ & $\mathrm{X}$ & $\mathrm{L}$ & $\mathrm{L}$ & $\mathrm{L}$ & $\mathrm{X}$ & $\mathrm{L}$ & Kanlayavattanakul et al., 2017 \\
\hline $\mathrm{X}$ & $\mathrm{X}$ & $\mathrm{L}$ & $\mathrm{L}$ & $\mathrm{H}$ & $\mathrm{L}$ & $\mathrm{L}$ & Milani and Sparavigna, 2017 \\
\hline $\mathrm{L}$ & $\mathrm{L}$ & $\mathrm{L}$ & $\mathrm{L}$ & $\mathrm{L}$ & $\mathrm{L}$ & $\mathrm{L}$ & Srivilai et al., 2017 \\
\hline $\mathrm{X}$ & $\mathrm{X}$ & $\mathrm{L}$ & $\mathrm{L}$ & $\mathrm{L}$ & $\mathrm{L}$ & $\mathrm{L}$ & Wang et al., 2018 \\
\hline $\mathrm{X}$ & $\mathrm{X}$ & $\mathrm{L}$ & $\mathrm{H}$ & $\mathrm{L}$ & $\mathrm{L}$ & $\mathrm{L}$ & Egoumenides et al., 2018 \\
\hline $\mathrm{X}$ & $\mathrm{X}$ & $\mathrm{L}$ & $\mathrm{L}$ & $\mathrm{H}$ & $\mathrm{H}$ & $\mathrm{L}$ & Goldberg et al., 2019 \\
\hline $\mathrm{X}$ & $\mathrm{X}$ & $\mathrm{L}$ & $\mathrm{L}$ & $\mathrm{L}$ & $\mathrm{L}$ & $\mathrm{X}$ & Lee and Cha, 2018 \\
\hline $\mathrm{L}$ & $\mathrm{L}$ & $\mathrm{L}$ & $\mathrm{L}$ & $\mathrm{L}$ & $\mathrm{L}$ & $\mathrm{L}$ & Matsuyama et al., 2018 \\
\hline $\mathrm{L}$ & $\mathrm{X}$ & $\mathrm{X}$ & $\mathrm{L}$ & $\mathrm{L}$ & $\mathrm{X}$ & $\mathrm{L}$ & Weber et al., 2019 \\
\hline $\mathrm{X}$ & $\mathrm{X}$ & $\mathrm{L}$ & $\mathrm{L}$ & $\mathrm{L}$ & $\mathrm{L}$ & $\mathrm{L}$ & Yoshida et al., 2019 \\
\hline $\mathrm{X}$ & $\mathrm{L}$ & $\mathrm{L}$ & $\mathrm{H}$ & $\mathrm{L}$ & $\mathrm{L}$ & $\mathrm{L}$ & Asada et al., 2019 \\
\hline $\mathrm{L}$ & $\mathrm{L}$ & $\mathrm{L}$ & $\mathrm{L}$ & $\mathrm{L}$ & $\mathrm{L}$ & $\mathrm{L}$ & Roh et al., 2019 \\
\hline
\end{tabular}




\begin{tabular}{|c|c|c|c|c|c|c|c|}
\hline \multicolumn{2}{|c|}{ Selection bias } & \multirow{2}{*}{$\begin{array}{c}\text { Reporting bias } \\
\begin{array}{c}\text { Selective } \\
\text { reporting }\end{array}\end{array}$} & \multirow{2}{*}{$\begin{array}{c}\text { Other biases } \\
\begin{array}{c}\text { Other sources } \\
\text { of bias }\end{array}\end{array}$} & \multirow{2}{*}{$\begin{array}{c}\text { Performance bias } \\
\text { Blinding } \\
\text { (participants and } \\
\text { personnel) }\end{array}$} & \multirow{2}{*}{$\begin{array}{c}\text { Detection bias } \\
\text { Blinding } \\
\text { (outcome } \\
\text { assessment) }\end{array}$} & \multirow{2}{*}{$\begin{array}{c}\text { Attrition bias } \\
\begin{array}{c}\text { Incomplete } \\
\text { outcome data }\end{array}\end{array}$} & \\
\hline $\begin{array}{c}\text { Random sequence } \\
\text { generation }\end{array}$ & $\begin{array}{c}\text { Allocation } \\
\text { concealment }\end{array}$ & & & & & & \\
\hline $\mathrm{X}$ & $\mathrm{X}$ & $\mathrm{L}$ & $\mathrm{L}$ & $\mathrm{L}$ & $\mathrm{L}$ & $\mathrm{L}$ & Zeng et al., 2019 \\
\hline $\mathrm{L}$ & $\mathrm{X}$ & $\mathrm{L}$ & $\mathrm{L}$ & $\mathrm{L}$ & $\mathrm{L}$ & $\mathrm{L}$ & Zhang et al., 2019 \\
\hline $\mathrm{X}$ & $\mathrm{X}$ & $\mathrm{L}$ & $\mathrm{L}$ & $\mathrm{L}$ & $\mathrm{H}$ & $\mathrm{X}$ & Ahmad et al., 2020 \\
\hline $\mathrm{L}$ & $\mathrm{X}$ & $\mathrm{L}$ & $\mathrm{L}$ & $\mathrm{L}$ & $\mathrm{L}$ & $\mathrm{L}$ & de Toledo Bagatin et al., 2020 \\
\hline \multirow[t]{2}{*}{$\mathrm{X}$} & $\mathrm{X}$ & $\mathrm{L}$ & $\mathrm{L}$ & $\mathrm{L}$ & $\mathrm{L}$ & $\mathrm{L}$ & Im et al., 2020 \\
\hline & & & Skin care RCTs & & & & \\
\hline $\mathrm{X}$ & $\mathrm{X}$ & $\mathrm{L}$ & $\mathrm{L}$ & $\mathrm{L}$ & $\mathrm{L}$ & $\mathrm{H}$ & Hay, 1998 \\
\hline $\mathrm{L}$ & $\mathrm{X}$ & $\mathrm{L}$ & $\mathrm{X}$ & $\mathrm{L}$ & $\mathrm{L}$ & $\mathrm{L}$ & Kamimura et al., 2000 \\
\hline $\mathrm{X}$ & $\mathrm{X}$ & $\mathrm{L}$ & $\mathrm{X}$ & $\mathrm{H}$ & $\mathrm{L}$ & $\mathrm{L}$ & Satchell et al., 2002 \\
\hline $\mathrm{X}$ & $\mathrm{X}$ & $\mathrm{L}$ & $\mathrm{X}$ & $\mathrm{L}$ & $\mathrm{L}$ & $\mathrm{L}$ & Herrera-Arellano et al., 2004 \\
\hline $\mathrm{X}$ & $\mathrm{X}$ & $\mathrm{L}$ & $\mathrm{L}$ & $\mathrm{H}$ & $\mathrm{H}$ & $\mathrm{L}$ & Sasmaz and Arican, 2005 \\
\hline $\mathrm{X}$ & $\mathrm{X}$ & $\mathrm{H}$ & $\mathrm{X}$ & $\mathrm{H}$ & $\mathrm{H}$ & $\mathrm{X}$ & Salmanpoor et al., 2012 \\
\hline $\mathrm{L}$ & $\mathrm{X}$ & $\mathrm{L}$ & $\mathrm{L}$ & $\mathrm{L}$ & $\mathrm{L}$ & $\mathrm{L}$ & Choi et al., 2015 \\
\hline $\mathrm{X}$ & $\mathrm{X}$ & $\mathrm{L}$ & $\mathrm{X}$ & $\mathrm{L}$ & $\mathrm{L}$ & $\mathrm{L}$ & FAAD, 2018 \\
\hline $\mathrm{L}$ & $\mathrm{L}$ & $\mathrm{L}$ & $\mathrm{L}$ & $\mathrm{L}$ & $\mathrm{L}$ & $\mathrm{X}$ & Chaijan et al., 2018 \\
\hline $\mathrm{L}$ & $\mathrm{X}$ & $\mathrm{L}$ & $\mathrm{L}$ & $\mathrm{L}$ & $\mathrm{H}$ & $\mathrm{L}$ & Pekmezci et al., 2018 \\
\hline
\end{tabular}

$\mathrm{L}=$ Low risk; $\mathrm{H}=$ High risk; $\mathrm{X}=$ Unclear risk.

Table 4. Plants and/or herbs found effective in the identified RCTs and available in Jordan.

\begin{tabular}{cc}
\hline Plant/herbs available in Jordan & aAvailability in Jordan based on the city \\
\hline A. sativa & Irbid \\
C. siliqua & Petra and Tal Al-Rumman \\
F. vulgare & Madaba and Tal Al-Rumman \\
G. glabra & Jordan bridge \\
M. sativa & Al-Mafraq and Ain Al Beida \\
M. communis & Karak Governorate and Alkaser \\
Olea europaea & Amman, Tal Al-Rumman and Wadi Orjan \\
P. oleracea & Al-Mafraq, Ajloun, Amman, Aqaba, and Moab \\
S. marianum & Irbid and outskirt of Irbid \\
U. urens & Tal Al-Rumman \\
\hline
\end{tabular}

aAvailability in Jordan as listed in "The Plants of Jordan: An Annotated Checklist."

(Matsuyama et al., 2018); and the third study tested Sphaeranthus indicus for its moisturizing, antihyperpigmentation, antisebum, elasticity properties, and overall improvement of the skin condition (Ahmad et al., 2020).

\section{Hair care}

Ten RCTs concerned with hair care were identified for reducing hair loss and baldness, six of which were published between 1998 and 2018. The studies used O. sativa (Choi et al., 2015), Malus pumila (Kamimura et al., 2000), and anthralin (Sasmaz and Arican, 2005), and three used a combination of natural products. Of those combinations, one study used Thymus vulgaris, Lavandula angustifolia, Rosmarinus officinalis, and Cedrus atlantica (Hay, 1998); another study used Matricaria chamomilla, Achillea millefolium, Ceratonia siliqua, Equisetum arvense, Urtica urens, and Urtica dioica (Pekmezci et al., 2018), whereas the third one used Ashwagandha, curcumin, Saw palmetto, tocotrienol/tocopherol complex, piperine, capsaicin, hydrolyzed marine collagen, hyaluronic acid, and organic kelp (FAAD, 2018).

Four studies published between 2002 and 2018 tested natural products and compounds for their antidandruff activity; they used Melaleuca alternifolia (Satchell et al., 2002), Solanum chrysotrichum (Herrera-Arellano et al., 2004), G. glabra (Salmanpoor et al., 2012), and Myrtus communis (Chaijan et al., 2018).

\section{Risk of Bias of the Included Studies}

The risk of bias of the included studies in this review was conducted by the researchers, with the bias assessment for all the studies included in detail in Table 3.

\section{Skin care}

Eighteen studies were at low risk of randomization bias, as they were randomized using either sequence generation or block randomization. On the other hand, 3 studies were at a high risk due to inadequate randomization. The rest did not provide sufficient information regarding the randomization method; thus, the risk was considered unclear $(n=32)$. Most studies $(n=42)$ did not describe the allocation concealment process, so the risk of bias was noted as unclear, whereas 10 studies were considered of low risk as proper concealment techniques were described. Only one study was considered high risk regarding the allocation concealment, as the participants were unblinded. Fifty-two other studies were considered of low-risk selective reporting bias while only one was considered unclear, as the $p$ values were not reported in the results section (Weber et al., 2019). Many studies $(n=39)$ were double-blinded and therefore at a low risk of performance and detection biases. The participants were not blinded in 9 studies, so these were considered at a high risk of performance bias. Additionally, the assessors were not blinded in 6 studies, so these were at a high risk of detection bias. If it was unclear whether 
the participants and/or assessors were blinded, the studies were considered to be at an unclear risk of performance/detection bias. For the attrition bias, attrition rates above $20 \%$ were considered of high risk; 2 studies were found to have high dropout rates and were at a high risk of attrition bias, 43 were stated as low risk, and 8 were of unclear risk because it was unclear whether the data of all participants were considered or if any failed to complete the study. Many studies $(n=43)$ were stated as low risk in the domain of other biases, six were stated as high risk as the researchers were funded from the same companies providing the test products, and eight were considered unclear because the conflict of interest was not declared in these studies.

\section{Hair care}

Four studies were at low risk of randomization bias, while six were considered of unclear risk, because the randomization methods were not mentioned. One study was considered at low risk of allocation bias, and nine were considered of unclear risk. In the reporting bias domain, nine were considered low risk and one was of unclear risk. Some studies $(n=7)$ were double-blinded, so they were at low risk of both performance and detection biases, while the remaining three studies followed an open-label strategy and thus were at a high risk for both performance and detection biases. In the attrition bias domain, seven studies were at low risk, two were at an unclear risk, and one was stated high risk. Regarding other biases, five studies were considered low risk, and the other five were stated as unclear risk.

\section{Outcomes}

Skin care

Thirty-six RCTs compared natural product(s) or derived compound(s) with a placebo; 29 preparations were found effective. Nine RCTs compared natural product(s) or derived compound(s) with another treatment; eight preparations were found effective. Eight RCTs compared natural product(s) or derived compound(s) with untreated controls, all of which yielded statistically significant results. One RCT compared silymarin cream with untreated control and placebo was found effective in both cases. The results of the remaining studies were statistically insignificant.

\section{Hair care}

Six RCTs compared natural product(s) or derived compound(s) to placebo, and all were found to have statistically significant outcomes. Four RCTs compared natural product(s) or derived compound(s) with active treatments, and the efficacy outcomes were found to be statistically insignificant.

The tested natural product(s) or derived compound(s) showed good tolerability in most studies, but adverse events (AEs) including dryness, scaling, erythema, edema, itching, and pricking were reported with a formulation containing retinol and rose extract (Lee et al., 2011).

\section{Plants present in Jordan}

From the above discussed studies, ten plants were identified to be present in different locations in Jordan, as illustrated in Table 4. The plants that are found in Jordan and can be used for skin and hair care were as follows: A. sativa, which was investigated in a study conducted in the United States of America (USA), was found effective in improving skin barrier integrity and increasing its hydration (Garay, 2016). Portulaca oleracea was reported to be useful in the treatment of melasma in China (Zhang et al., 2019). In addition, it was found to improve skin health and reduce sensitization when used in combination with other plants (Wang et al., 2018). Silybum marianum was also reported to be useful for melasma as was found in an Iraqi study (Altaei, 2012). Foeniculum vulgare and M. sativa were effective in reducing facial hair in two studies, both conducted in Iran (Javidnia et al., 2003; Sargazi et al., 2016). Glycyrrhiza glabra was used in combination with other plants to improve the overall skin condition (Roh et al., 2019; Seiwerth et al., 2019) and in a hair shampoo as an antidandruff agent (Salmanpoor et al., 2012). Myrtus communis was also found useful for the treatment of dandruff when mixed with vinegar in a study conducted in Iran (Chaijan et al., 2018). Ceratonia siliqua and U. urens were used in a combination used to improve hair growth and strength with other plants in a study conducted in Turkey (Pekmezci et al., 2018).

\section{DISCUSSION}

The worldwide growth of the cosmetic sector is partially driven by the input of natural products (Cervellon and Carey, 2011). The global market value for natural cosmetics is expecting a positive increase with the upcoming years (Shahbandeh, 2020). This SR is unique as it explored plant extracts, herbal preparations, and isolated plant-derived compounds used for cosmetic purposes, particularly for skin and hair care. Fiftythree RCTs exploring natural products used for skin care were identified. Most of the RCTs were published between the years 2013 and $2019(n=34)$. Thirty-seven RCTs used natural products as a single ingredient (around $70 \%$ of the RCTs), while 16 RCTs used them in combinations (around 30\% of the RCTs). Creams were the most frequently used dosage form (49\% of the topical preparations). Other tested dosage forms were lotions, serums, gels, emulsions, beverages, oils, tablets, and capsules. Ten RCTs were designed to test natural products for hair care, seven of which used natural products as a single ingredient $(70 \%$ of the RCTs), while the other three used them in combinations (30\% of the RCTs). Shampoos were the most common form used (40\%); aromatic oils, creams, solutions, capsules, and hair tonics were also used. The population included in this review added up to 3,439 subjects, and about $50 \%$ of the studies included females exclusively. The smallest number of participants was 3 and the highest was 203. The most common duration of the RCTs was 12 weeks (around $27 \%$ of the RCTs). The shortest duration was 75 minutes ( 0.0074 weeks), and the longest duration was 7 months (30.42 weeks). Around $87.3 \%$ of the reviewed RCTs found the tested natural products to have statistically significant efficacy outcomes. In addition, several treatment preparations were described, highlighting the diverse possibilities for incorporating botanicals into cosmetics. However, these reported outcomes should be interpreted cautiously for several reasons. Firstly, the majority of RCTs did not include power calculations indicating whether the number of participants is representative of the population or not. Secondly, participants were usually from a certain background, and thus the findings might not be applicable to all people of diverse ethnicities, lacking external generalizability. 
Thirdly, statistical significance may not necessarily indicate clinical significance, as the outcomes of interest are sometimes surrogate markers. It is important to explore local plants for their cosmetic potential, which may add to the diversity of the local market. In our case, plants that are present in Jordan are of particular interest, as these can be used in developing cosmetics locally and can be marketed internationally to help the country's economy flourish (Workman, 2020). This discussion sheds light on each of these plants to provide needed information for policy makers and investors in the country.

\section{CONCLUSION}

Cosmetics are marketed and used worldwide for various purposes, which makes them a subject for academic and market research (Infante et al., 2016). This SR provided a summary of the plants/herbs in the literature which were clinically tested in RCTs from 1997 until 2020 for their cosmetic purposes, particularly skin and hair care. Such information can be helpful for policy makers and investors to make informed decisions regarding the production of cosmetics that can be of benefit locally and internationally. Additionally, this SR provided a list of plants/herbs found in Jordan which evidently showed cosmetic potential. In order to provide clear and comparative results, plants with promising findings are worth further investigations in robust RCTs.

\section{AUTHORS' CONTRIBUTION}

All authors made substantial contributions to conception and design, acquisition of data, or analysis and interpretation of data; took part in drafting the article or revising it critically for important intellectual content; agreed to submit to the current journal; gave final approval of the version to be published; and agreed to be accountable for all aspects of the work.

\section{ETHICAL APPROVAL}

Not applicable.

\section{CONFLICT OF INTEREST}

The authors report no financial or any other conflicts of interest in this work.

\section{FUNDING}

There is no funding to report.

\section{REFERENCES}

Aali N, Sargolzaeiaval F, Sepehri Z. Effect of Alfalfa (Medicago sativa L.) extract on undesired hair growth in human: a safe remedy for cosmetic procedures. Der Pharm Lett, 2016; 8(19):351-6.

Aburjai T, Hudaib M, Tayyem R, Yousef M, Qishawi M. Ethnopharmacological survey of medicinal herbs in Jordan, the Ajloun Heights region. J Ethnopharmacol, 2007; 110(2):294-304.

Afifi FU, Abu-Irmaileh B. Herbal medicine in Jordan with special emphasis on less commonly used medicinal herbs. J Ethnopharmacol, 2000; 72(1-2):101-10.

Ahmad HI, Khan HMS, Akhtar N. Development of topical drug delivery system with Sphaeranthus indicus flower extract and its investigation on skin as a cosmeceutical product. J Cosmet Dermatol, 2020; 19(4):985-94.

Akhtar N, Ijaz S, Khan HMS, Uzair B, Reich A, Khan BA. Ziziphus mauritiana leaf extract emulsion for skin rejuvenation. Trop J Pharm Res, 2016; 15(5):929-36.
Akhtar NA, Zaman SU, Khan BA, Amir MN, Ebrahimzadeh MA. Calendula extract effects on mechanical parameters of human skin. Acta Pol Pharm, 2011; 68(5):693-701.

Altaei $\mathrm{T}$. The treatment of melasma by silymarin cream. BMC Dermatol, 2012; 12(1):1-6.

Alzweiri M, Sarhan AA, Mansi K, Hudaib M, Aburjai T. Ethnopharmacological survey of medicinal herbs in Jordan, the Northern Badia region. J Ethnopharmacol, 2011; 137(1):27-35.

Antignac E, Nohynek GJ, Re T, Clouzeau J, Toutain H. Safety of botanical ingredients in personal care products/cosmetics. Food Chem Toxicol, 2011; 49(2):324-41.

Asada K, Ohara T, Muroyama K, Yamamoto Y, Murosaki S. Effects of hot water extract of Curcuma longa on human epidermal keratinocytes in vitro and skin conditions in healthy participants: a randomized, double-blind, placebo-controlled trial. J Cosmet Dermatol, 2019; 18(6):1866-74.

Ashawat M, Banchhor M, Saraf S, Saraf S. Herbal cosmetics: "trends in skin care formulation". Pharmacogn Rev, 2009; 3(5):82-9.

Bauza E, Dal Farra C, Berghi A, Oberto G, Peyronel D, Domloge N. Date palm kernel extract exhibits antiaging properties and significantly reduces skin wrinkles. Int J Tissue React, 2002; 24(4):131.

Bazin R, Flament F, Colonna A, Le Harzic R, Buckle R, Piot B, Laizé F, Kaatz M, König K, Fluhr JW. Clinical study on the effects of a cosmetic product on dermal extracellular matrix components using a highresolution multiphoton tomograph. Skin Res Technol, 2010; 16(3):305-10.

Boonchai W, Varothai S, Winayanuwattikun W, Phaitoonvatanakij S, Chaweekulrat P, Kasemsarn P. Randomized investigator-blinded comparative study of moisturizer containing 4-t-butylcyclohexanol and licochalcone A versus $0.02 \%$ triamcinolone acetonide cream in facial dermatitis. J Cosmet Dermatol, 2018; 17(6):1130-5.

Braithwaite I, Hunt A, Riley J, Fingleton J, Kocks J, Corin A, Helm C, Sheahan D, Tofield C, Montgomery B, Holliday M, Weatherall M, Beasley R. Randomised controlled trial of topical kanuka honey for the treatment of rosacea. BMJ Open, 2015; 5(6):e007651.

Camouse MM, Domingo DS, Swain FR, Conrad EP, Matsui MS, Maes D, Declercq L, Cooper KD, Stevens SR, Baron ED. Topical application of green and white tea extracts provides protection from solarsimulated ultraviolet light in human skin. Exp Dermatol, 2009; 18(6):522-6.

Cervellon MC, Carey L. Consumers' perceptions of "green": why and how consumers use eco-fashion and green beauty products. Crit Stud Fashion Beauty, 2011; 2(1):117-38.

Chaijan MR, Handjani F, Zarshenas M, Rahimabadi MS, Tavakkoli A. The Myrtus communis L. solution versus ketoconazole shampoo in treatment of dandruff A double blinded randomized clinical trial. J Pak Med Assoc, 2018; 68(5):715-20.

Choi JS, Park JB, Moon WS, Moon JN, Son SW, Kim MR. Safety and efficacy of rice bran supercritical $\mathrm{CO} 2$ extract for hair growth in androgenic alopecia: a 16-week double-blind randomized controlled trial. Biol Pharm Bull, 2015; 38(12):1856-63.

Danby SG, AlEnezi T, Sultan A, Lavender T, Chittock J, Brown K, Cork MJ. Effect of olive and sunflower seed oil on the adult skin barrier: implications for neonatal skin care. Pediatr Dermatol, 2013; 30(1):42-50.

de Toledo Bagatin J, Bagatin E, Campos PMBGM. A pilot clinical study to evaluate the effectiveness of olive extract containing hydroxytyrosol for oral and topical treatment of melasma. Biomed Pharmacol J, 2020; 17(1):1-15.

Domzal T, Kernan J. Variations on the pursuit of beauty: toward a corporal theory of the body. Psychol Mark, 1993; 10(6):495-511.

Egoumenides L, Gauthier A, Barial S, Saby M, Orechenkoff C, Simoneau G, Carillon J. A specific melon concentrate exhibits photoprotective effects from antioxidant activity in healthy adults. Nutrients, 2018; 10(4):437.

FAAD GAM. A six-month, randomized, double-blind, placebocontrolled study evaluating the safety and efficacy of a nutraceutical supplement for promoting hair growth in women with self-perceived thinning hair. J Drugs Dermatol, 2018; 17(5):558-65. 
Filipovic M, Gledovic A, Lukic M, Tasic-Kostov M, Isailovic $\mathrm{T}$, Pantelic I, et al. Alp rose stem cells, olive oil squalene and a natural alkyl polyglucoside emulsifier: are they appropriate ingredients of skin moisturizers - in vivo efficacy on normal and sodium lauryl sulfate irritated skin? Vojnosanit Pregl, 2016; 73(11):991-1002.

Fonseca-Santos B, Corrêa MA, Chorilli M. Sustainability, natural and organic cosmetics: consumer, products, efficacy, toxicological and regulatory considerations. Braz J Pharm Sci, 2015; 51(1):17-26.

Garay M. Colloidal oatmeal (Avena sativa) improves skin barrier through multi-therapy activity. J Drugs Dermatol, 2016; 15(6):684-90.

Gediya S, Mistry R, Patel U, Blessy M, Jain HN. Herbal plants used as a cosmetics. J Nat Prod Plant Resour, 2011; 1(1):24-32.

Goldberg DJ, Robinson DM, Granger C. Clinical evidence of the efficacy and safety of a new 3-in-1 anti-aging topical night serum-inoil containing melatonin, bakuchiol, and ascorbyl tetraisopalmitate: 103 females treated from 28 to 84 days. J Cosmet Dermatol, 2019; 18(3):806-14.

Gonzalez S, Pathak MA, Cuevas J, Villarrubia VG, Fitzpatrick TB. Topical or oral administration with an extract of Polypodium leucotomos prevents acute sunburn and psoralen-induced phototoxic reactions as well as depletion of Langerhans cells in human skin. Photodermatol Photoimmunol Photomed, 1997; 13(1-2):50-60.

Hay IC, Jamieson M, Ormerod AD. Randomized trial of aromatherapy: successful treatment for alopecia areata. Arch Dermatol, 1998; 134(11):1349-52.

Herrera-Arellano A, Jiménez-Ferrer E, Vega-Pimentel AM, de los Ángeles Martínez-Rivera M, Hernández-Hernández M, Zamilpa A, Tortoriello J. Clinical and mycological evaluation of therapeutic effectiveness of Solanum chrysotrichum standardized extract on patients with Pityriasis capitis (dandruff). A double blind and randomized clinical trial controlled with ketoconazole. Planta Med, 2004; 70(6):483-8.

Higgins JP, Altman DG, Gotzsche PC, Juni P, Moher D, Oxman AD, Savovic J, Schulz KF, Weeks L, Sterne JAC, Cochrane Bias Methods Group, Cochrane Statistical Methods Group. The Cochrane Collaboration's tool for assessing risk of bias in randomised trials. BMJ, 2011; 343:d5928.

Hosnuter M, Payasli C, Isikdemir A, Tekerekoglu B. The effects of onion extract on hypertrophic and keloid scars. J Wound Care, 2007; 16(6):251-4.

Ilnytska O, Kaur S, Chon S, Reynertson KA, Nebus J, Garay M, Mahmood K, Southall M. Colloidal oatmeal (Avena Sativa) improves skin barrier through multi-therapy activity. J Drugs Dermatol, 2016; 15(6):684 90

Im AR, Nam J, Ji KY, Cha S, Yoon J, Seo YK, Chae S, Kimb JY. Wrinkle reduction using a topical herbal cream in subjects classified by Sasang constitutional medicine as Soyang type: a randomized double-blind placebo-controlled study. Eur J Integr Med, 2020; 35:101070.

Infante VHP, Calixto LS, Campos PMBGM. Cosmetics consumption behaviour among men and women and the importance in products indication and treatment adherence. Surg Cosmet Dermatol, 2016; $8(2): 134-41$

Javidnia K, Dastgheib L, Mohammadi Samani S, Nasiri A. Antihirsutism activity of fennel (fruits of Foeniculum vulgare) extract. A double-blind placebo controlled study. Phytomedicine, 2003; 10(6-7):455-8.

Kamimura A, Takahashi T, Watanabe Y. Investigation of topical application of procyanidin B-2 from apple to identify its potential use as a hair growing agent. Phytomedicine, 2000; 7(6):529-36.

Kanlayavattanakul M, Fungpaisalpong K, Pumcharoen M, Lourith N. Preparation and efficacy assessment of malva nut polysaccharide for skin hydrating products. Ann Pharm Fr, 2017; 75(6):436-45.

Kanlayavattanakul M, Lourith N, Chaikul P. Jasmine rice panicle: a safe and efficient natural ingredient for skin aging treatments. J Ethnopharmacol, 2016, 4(193):607-16.

Kim S, Seock YK. Impacts of health and environmental consciousness on young female consumers' attitude towards and purchase of natural beauty products. Int J Consum Stud, 2009; 33(6):627-38.

Kim YH, Chung CB, Kim JG, Ko KI, Park SH, Kim JH, Eom SY, Kim YS, Hwang YI, Kim KH. Anti-wrinkle activity of ziyuglycoside I isolated from a Sanguisorba officinalis root extract and its application as a cosmeceutical ingredient. Biosci Biotechnol Biochem, 2008; 72(2):303-11.

Kim YH, Kim KH, Han CS, Yang HC, Park SH, Jang HI, Kim JW, Choi YS, Lee NH. Anti-wrinkle activity of Platycarya strobilacea extract and its application as a cosmeceutical ingredient. J Cosmet Sci, 2010; 6(3):211

Laroche M, Bergeron J, Barbaro-Forleo G. Targeting consumers who are willing to pay more for environmentally friendly products. J Consum Mark, 2001; 18(6):503-20.

Lee H, Cha HJ. Poria cocos wolf extracts represses pigmentation in vitro and in vivo. Cell Mol Biol, 2018; 64(5): 80-4.

Lee HE, Ko JY, Kim YH, Yoo SR, Moon SH, Kim NI, Park CW, Kim JH, Koh HJ, Park WS, Ro YS. A double-blind randomized controlled comparison of APDDR-0901, a novel cosmeceutical formulation, and $0.1 \%$ adapalene gel in the treatment of mild-to-moderate acne vulgaris. Eur J Dermatol, 2011; 21(6):959-65.

Lee KE, Park JE, Jung E, Ryu J, Kim YJ, Youm JK, Kang S. A study of facial wrinkles improvement effect of veratric acid from cauliflower mushroom through photo-protective mechanisms against UVB irradiation. Arch Dermatol Res, 2016; 308(3):183-92.

Mandawgade SD, Patravale VB. Formulation and evaluation of exotic fat based cosmeceuticals for skin repair. Indian J Pharm Sci, 2008; 70(4):539-42

Martelli L, Berardesca E, Martelli M. Topical formulation of a new plant extract complex with refirming properties. Clinical and non-invasive evaluation in a double-blind trial. Int J Cosmet Sci, 2000; 22(3):201-6.

Matsuyama A, Kikuchi M, Shimoda H. Effect on skin condition by 8 -week ingestion of standardized cherry blossom flower extract (sakura extract-P). Int J Biomed Sci, 2018; 14(1):12-9.

Mendoza CG, Singzon IA, Handog EB. A randomized, doubleblind, placebo-controlled clinical trial on the efficacy and safety of 3\% Rumex occidentalis cream versus $4 \%$ hydroquinone cream in the treatment of melasma among Filipinos. Int J Dermatol, 2014; 53(11):1412-6.

Michelle GM. Colloidal Oatmeal (Avena sativa) improves skin barrier through multi-therapy activity. J Drugs Dermatol, 2016; 15(6):684-90.

Milani M, Sparavigna A. The 24-hour skin hydration and barrier function effects of a hyaluronic $1 \%$, glycerin $5 \%$, and Centella asiatica stem cells extract moisturizing fluid: an intra-subject, randomized, assessorblinded study. Clin Cosmet Investig Dermatol, 2017; 10:311-5.

Moher D, Liberati A, Tetzlaff J, Altman DG, Group P. Preferred reporting items for systematic reviews and meta-analyses: the PRISMA statement. PLoS Med, 2009; 6(7):e1000097.

Morag M, Nawrot J, Siatkowski I, Adamski Z, Fedorowicz T, Dawid-Pac R, Urbanska M, Nowak G. A double-blind, placebo-controlled randomized trial of Serratulae quinquefoliae folium, a new source of betaarbutin, in selected skin hyperpigmentations. J Cosmet Dermatol, 2015; 14(3):185-90.

Muhammad KW, Akthar N, Bakhsh S, Caldeira EJ, Khan B. Skin lightening and sebum control efficacy of a cosmetic emulsion containing extract of tamarind seeds on Asian skin type. Latin Am J Pharm, 2015; $34: 570-5$

Pekmezci E, Dündar C, Türkoğlu M. A proprietary herbal extract against hair loss in androgenetic alopecia and telogen effluvium: a placebocontrolled, single-blind, clinical-instrumental study. Acta Dermatovenerol Alp Pannonica Adriat, 2018; 27(2):51-7.

Ray S, Belch J, Craigie AM, Khan F, Kennedy G, Hill A, Barton KL, Dawe RS, Ibbotson SH. Can antioxidant-rich blackcurrant juice drink consumption improve photoprotection against ultraviolet radiation? Br J Dermatol, 2016; 174(5):1101-3

Ribeiro A, Estanqueiro M, Oliveira M, Sousa Lobo J. Main benefits and applicability of plant extracts in skin care products. Cosmetics, 2015; 2(2):48-65.

Rival D, Bonnet S, Sohm B, Perrier E. A Hibiscus abelmoschus seed extract as a protective active ingredient to favour FGF-2 activity in skin. Int J Cosmet Sci, 2009; 31(6):419-26. 
Roh SS, Choi I, Kim HM, Lee MS, Jin MH, Kim BH, Hwang SJ, Kim MH. Clinical efficacy of herbal extract cream on the skin hydration, elasticity, thickness, and dermis density for aged skin: a randomized controlled double-blind study. J Cosmet Dermatol, 2019; 18(5):1389-94.

Salmanpoor R, Saki N, Mahmoodi G. Liquorice 7\% versus selenium sulfide $1 \%$ shampoos in the treatment of dandruff A clinical trial. Iran J Dermatol, 2012; 15(4):144-5.

Sargazi AAS, Nadakkavukaran P, Jim, Srgazi A, Aali N, Sargolzaeiaval F, Sepehri Z. Effect of Alfalfa (Medicago sativa L.) extract on undesired hair growth in human: a safe remedy for cosmetic procedures. Der Pharm Lett, 2016; 8(19):351-6.

Sasmaz S, Arican O. Comparison of azelaic acid and anthralin for the therapy of patchy alopecia areata. Am J Clin Dermatol, 2005; 6(6):403-6.

Satchell AC, Saurajen A, Bell C, Barnetson RS. Treatment of dandruff with $5 \%$ tea tree oil shampoo. J Am Acad Dermatol, 2002; 47(6):852-5.

Sawicka B, Noaema A. Cosmetics active ingredients used in the Middle East. Conference: II National Scientific Conference "Modern technologies and treatments in cosmetology". Lublin, Poland, 2015.

Seiwerth J, Tasiopoulou G, Hoffmann J, Wolfle U, Schwabe K, Quirin KW, Schempp CM. Anti-inflammatory effect of a novel topical herbal composition (VEL-091604) consisting of gentian root, licorice root and willow bark extract. Planta Med, 2019; 85(7):608-14.

Shahbandeh M. Global market value for natural and organic cosmetics and personal care from 2018 to 2027 [Online]. Statista. 2020 Available via https://www.statista.com/statistics/673641/global-marketvalue-for-natural-cosmetics/ (Accessed 23 October 2020).

Shilling C. Sociology and the body: classical traditions and new agendas. Sociol Rev, 2017; 55(1_Suppl):1-18.

Shin DH, Cha YJ, Joe GJ, Yang KE, Jang IS, Kim BH, Kim JM. Whitening effect of Sophora flavescens extract. Pharm Biol, 2013; 51(11):1467-76.

Shivanand P, Nilam M, Viral D. Herbs play an important role in the field of cosmetics. Int J Pharmtech Res, 2010; 2(1):632-9.

Soltanipour F, Delaram M, Taavoni S, Haghani H. The effect of olive oil and the $\mathrm{Saj}(\mathrm{R})$ cream in prevention of striae gravidarum: a randomized controlled clinical trial. Complement Ther Med, 2014; 22(2):220-5.

Srivilai J, Nontakhot K, Nutuan T, Waranuch N, Khorana $\mathrm{N}$, Wisuthiprot W, Scholfield $\mathrm{CN}$, Champachaisri K, Ingkaninan K. Sesquiterpene-enriched extract of Curcuma aeruginosa roxb. Retards axillary hair growth: a randomised, placebo-controlled, double-blind study. Skin Pharmacol Physiol, 2018; 31(2):99-106.

Srivilai J, Phimnuan P, Jaisabai J, Luangtoomma N, Waranuch N, Khorana N, Wisuitiprot W, Norman Scholfield C, Champachaisri K, Ingkaninan K. Curcuma aeruginosa Roxb. essential oil slows hairgrowth and lightens skin in axillae; a randomised, double blinded trial. Phytomedicine, 2017; 25:29-38.

Taifour H, El-Oqlah A, Ghazanfar S. The plants of Jordan: an annotated checklist. Kew Publishing, Richmond, London, 2017.

Tsuchiya T, Fukui Y, Izumi R, Numano K, Zeida M. Effects of oligomeric proanthocyanidins (OPCs) of red wine to improve skin whitening and moisturizing in healthy women-a placebo-controlled randomized double-blind parallel group comparative study. Eur Rev Med Pharmacol Sci, 2020; 24(3):1571-84.

Vicente RA, Leite e Silva VR, Baby AR, Velasco MV, Bedin V. Double-blind, randomized, placebo-controlled trial of a cream containing the Stryphnodendron adstringens (Martius) Coville bark extract for suppressing terminal hair growth. J Eur Acad Dermatol Venereol, 2009; 23(4):410-4.

Wang Y, Viennet C, Jeudy A, Fanian F, He L, Humbert P. Assessment of the efficacy of a new complex antisensitive skin cream. J Cosmet Dermatol, 2018; 17(6):1101-7.

Weber N, Schwabe K, Schempp CM, Wolfle U. Effect of a botanical cleansing lotion on skin sebum and erythema of the face: a randomized controlled blinded half-side comparison. J Cosmet Dermatol, $2019 ; 18(3): 821-6$.

White GM, Zhou HC, Burchette RJ. Biopsy followed by immediate curettage and electrodesiccation of suspected basal cell carcinomas at the first visit. JAMA Dermatol, 2013; 149(8):980-1.

Wineman E, Portugal-Cohen M, Soroka Y, Cohen D, Schlippe G, Voss W, Brenner S, Milner Y, Hai N, Ma'or Z. Photo-damage protective effect of two facial products, containing a unique complex of Dead Sea minerals and Himalayan actives. J Cosmet Dermatol, 2012; 11(3):183-92.

Workman D. Jordan's Top 10 exports. Jordan's Top 10 exports [Online]. World's Top Exports. 2020. Available via http://www. worldstopexports.com/jordans-top-10-exports/ (Accessed 24 October 2020).

Yoo S, Kim K, Nam H, Lee D. Discovering health benefits of phytochemicals with integrated analysis of the molecular network, chemical properties and ethnopharmacological evidence. Nutrients, 2018; 10(8):1052-60.

Yoshida H, Yamazaki K, Komiya A, Aoki M, Nakamura T, Kasamatsu S, Murata T, Sayo T, Okada Y, Takahashi Y. Inhibition of HYBID (KIAA1199)-mediated hyaluronan degradation and anti-wrinkle effect of Geranium thunbergii extract. J Cosmet Dermatol, 2019; 18(4):1052-60.

Zeng X, Li X, Wang X, Wen X, Jiang X. The effect of Zanthoxylum bungeanum maxim extract on crow's feet: a double-blind, split-face trial. Dermatol Ther, 2019; 32(6):e13079.

Zhang Q, Tu Y, Gu H, Sun D, Wu W, Man MQ, Chen H, Liu $\mathrm{H}, \mathrm{Ma} \mathrm{X}, \mathrm{He} \mathrm{L}$. A cream of herbal mixture to improve melasma. J Cosmet Dermatol, 2019; 18(6):1721-8.

Zhang YQ, Guan L, Zhong ZY, Chang M, Zhang DK, Li H, Lai W. The anti-inflammatory effect of cherry blossom extract (Prunus yedoensis) used in soothing skincare product. Int J Cosmet Sci, 2014 36(6):527-30.

How to cite this article:

Thiab S, Mhaidat NM, Taha MA, Thiab S, Koraysh S, Abutayeh R, Basheti I. A systematic review of randomized controlled trials assessing phytochemicals and natural ingredients for skin and hair care. J Appl Pharm Sci, 2021; 11(07):020-045. 